\title{
Variable structuring element based fuzzy morphological operations for single viewpoint omnidirectional images
}

\author{
Olivier Strauss, Frédéric Comby \\ LIRMM, UMR CNRS-Université Montpellier II, 161, rue Ada, \\ 34392 Montpellier Cedex 5, France \\ Tel: (+33) 467418578 - Fax: (+33) 467418500 \\ e-mail: Olivier.Strauss@lirmm.fr
}

\begin{abstract}
Morphological tools can provide transformations suitable for real projective images, but the camera and objects to be analyzed have to be positioned in such a manner that a regular mesh on the objects projects a regular mesh on the image. A morphological modification of the image is thus the projection of an equivalent operation on the object. Otherwise, due to perspective effects, a morphological operation on the image is not the projection of an equivalent operation on the objects to be analyzed. With catadioptric omnidirectional images, it is almost impossible to place the sensor such that a regular mesh on the scene projects a regular mesh on the image. Nevertheless, with proper calibration of a central catadioptric system, the projection of a regular structuring element in a scene can be determined for each point on the image.
\end{abstract}

The aim of this paper is to present new morphological operators that use this projective property. These operators make use of a structuring element of varying shape. Since this varying shape cannot be represented as a binary union of pixels, we propose to use a fuzzy extension of the classical grey-level morphology to account for this phenomenon. This fuzzy extension is performed via fuzzy integrals.

Keywords: omnidirectional images, single viewpoint, mathematical morphology, Choquet and Sugeno fuzzy integrals, standardized fuzzy partition, sampling, variable structuring element, filtering.

\section{Introduction}

Mathematical morphology is an image analysis and filtering tool that was developed by Jean Serra's team [1]. Set theory-based mathematical morphology involves comparing the image to be analyzed to a known geometrical unit, which is called a structuring element. This structuring element is moved in such a way that its origin covers all of the image positions, to highlight specific features.

Erosion and dilation are dual operations underlying mathematical morphology - whose name refers to the effects of each of these operators on a binary shape (Fig. 1). These operators can be combined to morphologically filter images, i.e. delete (or highlight) shapes on the image comparable to the structuring elements used. This filtering is involved in opening (erosion followed by dilation using the same structuring element) and closing (dilation followed by erosion using the same structuring element). Finally, the subjective edges of objects on an image can be highlighted on the basis of a morphological gradient, i.e. by comparing (or differentiating) an eroded image to a dilated image (using the same structuring element). We focused on these basic morphological tools in this study.
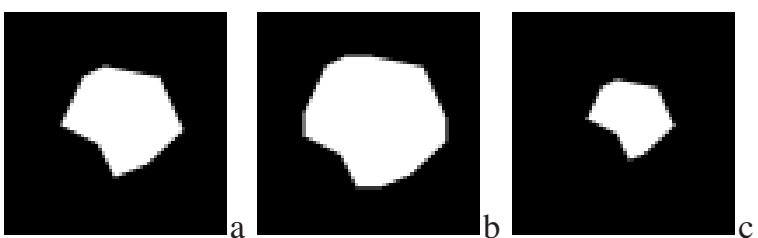

Figure 1: Binary (a) dilated (b) and eroded (c) images.

Mathematical morphology approaches were first developed for binary images, i.e. images derived from a classification. By these approaches, each pixel on a binary digital image is classified as belonging to one of the shapes to be analyzed or to the image background (thus a dual classification). This classification could be modified by including the area surrounding the structuring element in the morphological operation, in compliance with four basic properties [1]: translation invariance, bandwidth compatibility, local recognition and semicontinuity. These properties apply to discrete images, except for semicontinuity, which only applies in cases of continuous images.

Several extensions have been proposed to adapt, generalize or simply imitate morphological operations on grey-level images and functional structuring elements. However, there is some disagreement between authors concerning the designations of these extensions. We have adopted those put forward by Isabelle Bloch [2]. 
Generalization to grey-level images implies that the grey level of each pixel could be considered as a confidence measure indicating whether or not it belongs to the shape being analyzed. Similarly, the threshold for distinguishing the shape from the background is not very clear. The use of thresholds of decreasing values generates a series of nested binary images. The grey level could thus be considered as a functional parameter. This generalization is called grey-level morphology.

The so-called functional morphology generalization is implemented to model poor structuring element identification, or that the neighborhood which it defines cannot be represented as a union of image pixels. The structuring element could be considered as a kernel that serves as a confidence measure indicating whether or not a pixel belongs to the neighboring area of another pixel. Note that most functional morphologies are not internal - using a functional kernel on a binary image generally does not generate a binary image.

Finally, fuzzy morphology refers to a generalization whereby the image and structuring elements are considered as functional. Fuzzy morphology provides a suitable representation of the intuitive concept of fuzzy edges of objects and the arbitrary aspect of image partitioning (as a digital image is can be considered as resulting from the sampling and digitalization of a continuous image).

The morphological branch is distinguished from other image processing branches especially by the fact that the proposed solutions are simply discrete and not based on a continuous underlying illumination model. However, when the images are derived from image sensors (cameras), the efficacy of morphological tools in modifying or analyzing images with respect to the real scene of which they are a projection can be reduced if its projective nature is not taken into account.

The suitability of morphological tools for analyzing real projective images depends on the potential for positioning the sensor and the objects to be analyzed in such a manner that a regular mesh on the objects projects a regular mesh on the image. A morphological operation on the image, as defined with a regular image structuring element, is thus the projection of an equivalent morphological operation on the object.

When these conditions have not been fulfilled, due to perspective-induced resolution differences, a morphological modification of the image will not lead to an equivalent morphological modification of the object. Figure 2 illustrates that erosion does not uniformly alter the lines of a grid pattern.

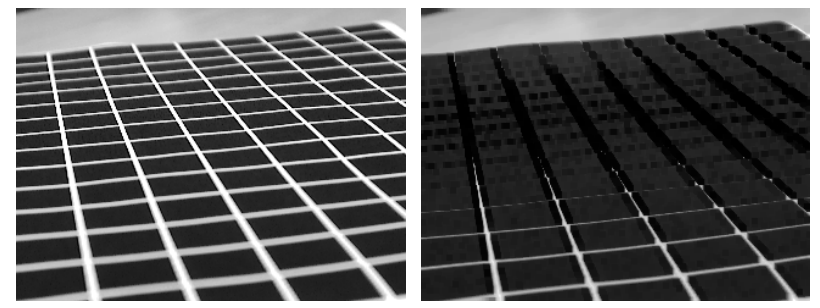

Figure 2: Erosion on a perspective image.

For omnidirectional images, it is almost impossible to place the sensor such that a regular grid on the scene is projected isotropically on the image (Fig. 3). However, single viewpoint omnidirectional sensors can be precalibrated in order to determine the projection of a regular structuring element over an entire perspective image reconstructed from an anamorphic omnidirectional image. This concept has already been used to estimate optical flow in an omnidirectional image sequence [3] and to facilitate matching of indices extracted from several omnidirectional images of the same scene [4]. However, to our knowledge, no studies have focused on using this concept for conventional filtering or morphological operations. Here we propose to apply this strategy to tailor morphological operators to grey-level omnidirectional images. These operators involve a variable-sized structuring element that thus cannot be expressed as a union of pixels. The morphology that we define can only be fuzzy. 


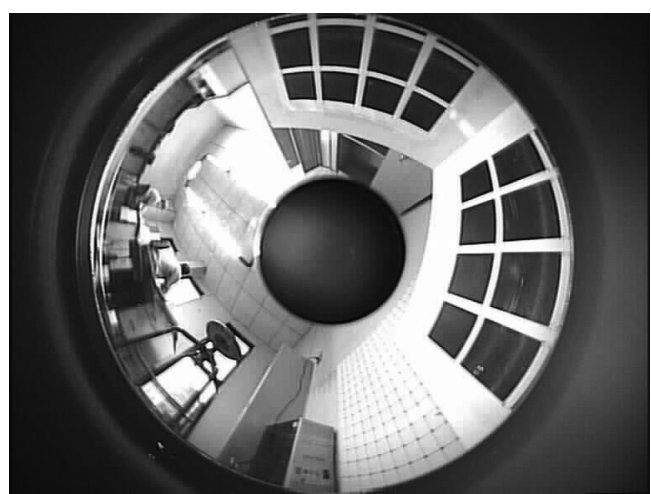

Figure 3: An omnidirectional image.

\section{Single viewpoint omnidirectional vision}

\subsection{Principle}

Cameras, like most perception systems, have a limited field of view - the perceived spatial range is limited by the size of the retina and the camera lens. It could be beneficial to broaden this field of view in many applications such as remote surveillance, videoconferencing, mobile robotics and inspection. Over the last 10 years or more, the vision community has been paying close attention to optical and mechanical processes that can broaden the field of view of cameras [5]. These processes could be divided into four categories.

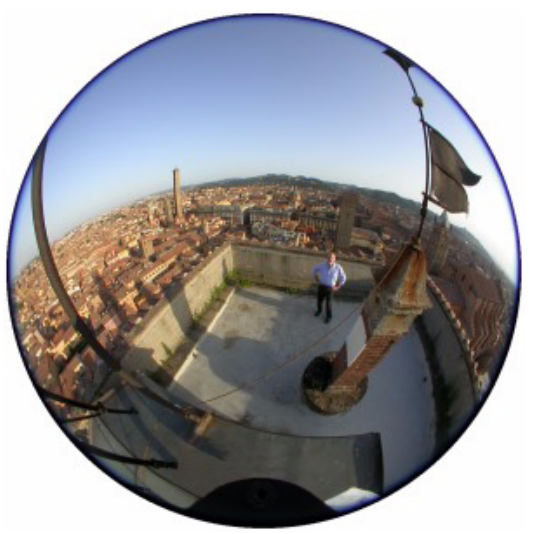

Figure 4: A fisheye image.

In the first process, a camera lens with a very short focal length is used instead of a standard lens. This socalled fisheye lens has an aperture angle of more than $180^{\circ}$. These lenses are complex to manufacture, so they are expensive, bulky and generate very deformed images (Fig. 4).

The second process involves rotating a standard camera around a fixed vertical axis. The set of different views obtained provides a panoramic image of the environment [6]. The advantage of this technique is that a normal camera can be used, but a very precise system must be set up to rotate the camera. Moreover, it takes quite a long time to capture a panoramic image and there can be no movement in the scene during the image acquisition period - which is a drawback for most applications. High esthetic quality images can, however, be obtained by this process (Fig. 5).

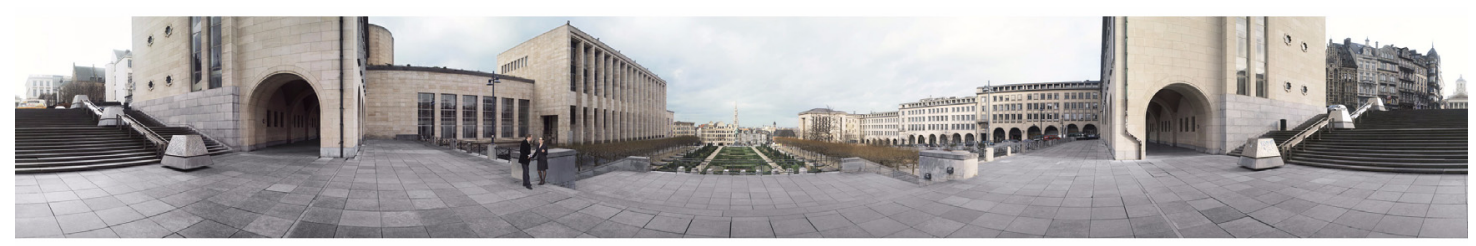

Figure 5: A rotational panoramic image.

Several stationary cameras with intersecting viewpoints can be used instead of a single rotating camera [7]. Images are captured from all the cameras synchronously, thus reducing the acquisition time. However, synchronized image acquisition is complex and expensive 
Currently the most popular solution is to use a standard camera combined with a reflecting lens. The lenses used have a plane layout or a surface of revolution $[8,9]$. In the latter case, as for fisheye lenses, an image viewed through an omnidirectional catadioptric sensor will be anamorphic (Fig. 3). A restoring process which is naturally called anti-anamorphosis - is required to correct images captured through such lenses for inspection or surveillance purposes.

For many applications, all images reconstructed from an omnidirectional image must be geometrically accurate and have the same viewpoint. Nayar and Baker's benchmark research [8] highlighted that few mirror shapes can overcome this constraint. Single viewpoint omnidirectional systems were the focus of an advanced study that gave rise to a very simple model which we adopted for this study.

\subsection{Fundamental equation for single viewpoint catadioptric cameras}

A single viewpoint catadioptric camera is, by definition, a system that maps a single point projected on the image to each point in the visible 3D space. Geyer and Daniilidis [10] found that this projection is mathematically equivalent to a two-step mapping via a sphere. This method, as illustrated in Figure 6, involves just two parameters $(\varphi$ and $\xi)$ to link a point $\mathrm{M}(\mathrm{X}, \mathrm{Y}, \mathrm{Z})$ with its projection $\mathrm{m}(\mathrm{x}, \mathrm{y})$ on the image:

$x=\frac{(\varphi+\xi) X}{\xi R-Z}, y=\frac{(\varphi+\xi) Y}{\xi R-Z}$,

where $\mathrm{R}^{2}=\mathrm{X}^{2}+\mathrm{Y}^{2}+\mathrm{Z}^{2}$.

Retina sampling results in the physical coordinates of $\mathrm{m}$ to its image coordinates $(\mathrm{u}, \mathrm{v})$ :

$\mathrm{u}=\mathrm{k}_{\mathrm{u}} \mathrm{x}+\mathrm{u}_{\mathrm{o}}, \mathrm{v}=\mathrm{k}_{\mathrm{v}} \mathrm{y}+\mathrm{v}_{\mathrm{o}}$,

where $\left(\mathrm{k}_{\mathrm{u}}, \mathrm{k}_{\mathrm{v}}, \mathrm{u}_{\mathrm{o}}, \mathrm{v}_{\mathrm{O}}\right)$ are constants of the image acquisition process. Combining equations (1) and (2) gives fundamental equations for single viewpoint catadioptric systems. In this model, only the coordinate change induced by sampling is taken into account

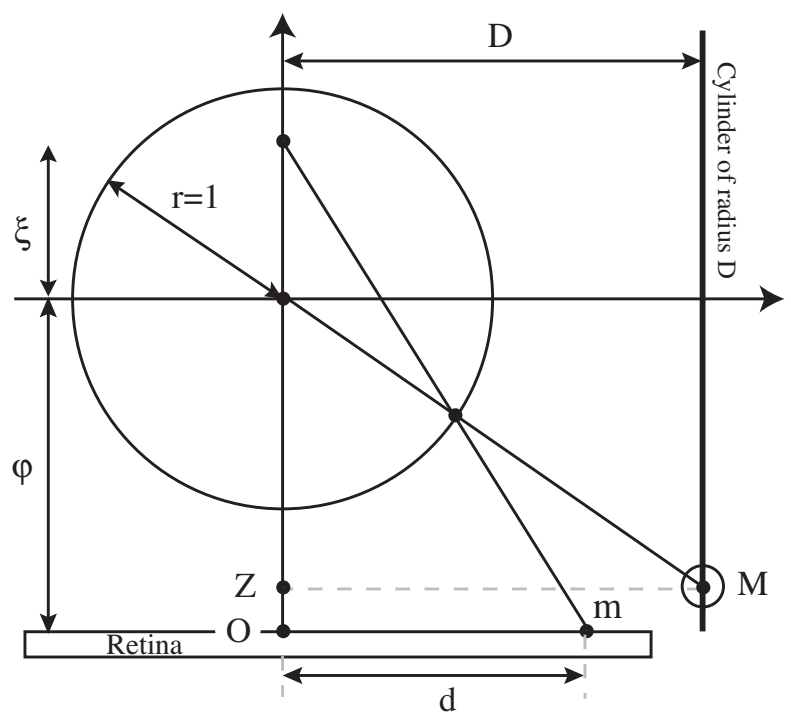

Figure 6: Double projection.

\subsection{Anti-anamorphosis of omnidirectional images}

Anamorphosis is an image distortion process that stifles the image's projective properties, while anti-anamorphosis is a reverse process that restores part or all of the image's projective properties, thus enabling interpretation. Single viewpoint omnidirectional catadioptric systems can reconstruct any perspective image within the sensor's field of view. This can theoretically be done by inversing equations (1) and (2) with point $\mathrm{M}$ becoming a member of the plane of the image that is being reconstructed.

In practice, sampling images created by this method gives irregular results. If we consider an omnidirectional image as a disc, the maximum projected resolution will be at the outer part of the disc, with the resolution quality decreasing on a gradient from the outer edge to the center of the image. To create a standard digital image, the best strategy is to define the mesh of the image to be reconstructed and then this mesh can be projected on the omnidirectional image. Interpolation techniques (variable structuring element if possi- 
ble) are thus used to estimate the grey level for each point of a reconstructed image (Fig. 7), but an infinite number of projective images corresponding to different viewpoints will be obtained for a single omnidirectional image.

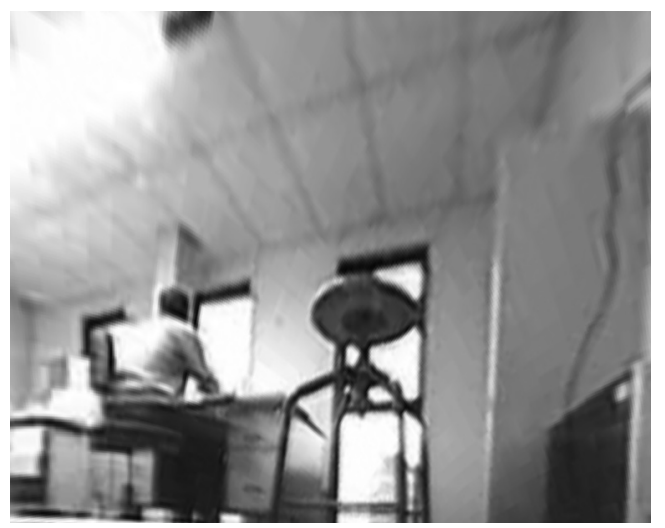

Figure 7: Anti-anamorphosis on an image plane.

To view an entire image, it is common to project the image on a cylinder surrounding the rotating mirror (Fig. 8). This projection encompasses all images that could be acquired by a camera revolving around an axis running through its projection reference point [6], which is the most common use of this type of sensor. The resulting image is easy to interpret but still distorted, i.e. straight lines running perpendicular to the mirror rotation axis are not projected as straight lines on the projected image.

All points $\mathrm{M}$ at coordinates $(\mathrm{X}, \mathrm{Y}, \mathrm{Z})$ on a cylinder of radius $\mathrm{D}$ are projected on the image plane at a point $\mathrm{m}$ at coordinates $(\mathrm{u}, \mathrm{v})$ :

$\mathrm{u}=\mathrm{k}_{\mathrm{u}} \mathrm{d} \cos (\theta)+\mathrm{u}_{0}, \mathrm{v}=\mathrm{k}_{\mathrm{v}} \mathrm{d} \sin (\theta)+\mathrm{v}_{0}$,

with $\mathrm{X}=\mathrm{D} \cos (\theta)$ and $\mathrm{Y}=\mathrm{D} \sin (\theta)$.

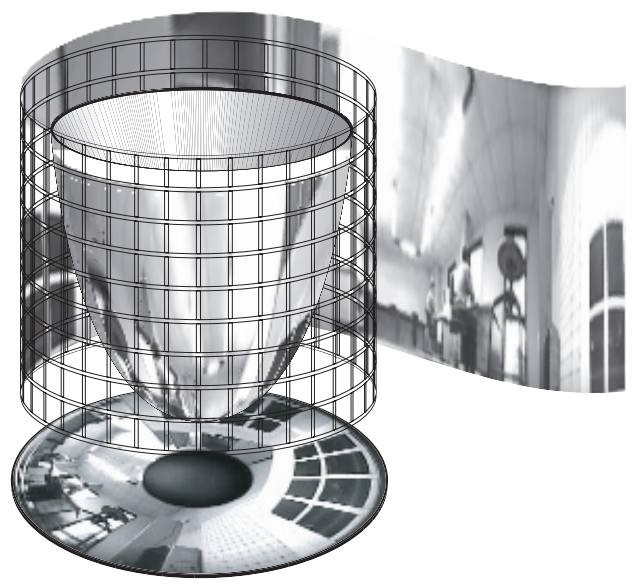

Figure 8: Projection cylinder.

Then, based on equation (1), with $\rho=Z / D$, gives:

$d=f(\rho)=\frac{(\varphi+\xi)}{\xi \sqrt{1+\rho^{2}}-\rho}$.

Anti-anamorphosis involves inversion of equation (4). Solving this problem (after eliminating erroneous solutions), gives:

$\rho=f^{-1}(d)=\frac{(\varphi+\xi)-\xi \sqrt{d^{2}\left(1-\xi^{2}\right)+(\varphi+\xi)^{2}}}{d\left(1-\xi^{2}\right)}$.

\subsection{Filtering anamorphic images}

Most omnidirectional image analysis studies use standard filtering operators. Such operators generally uti- 
lize the projective properties of standard images - a regular mesh on a surface that is almost perpendicular to the optical axis of the image sensor is projected as a regular mesh on the image. Projective transformation preserves the relative orientation of objects located on the same plane. An object is projected in an identical way, regardless of the relative position of the sensor, if the sensor and object maintain the same relative orientation [11].

When omnidirectional images are involved, the projective properties of the system cannot be used when anamorphosis has been caused by a mirror (or lens). In such cases, each image point seems to correspond to a different optical axis. For single viewpoint omnidirectional images, an image can be rectified through an anti-anamorphosis process. However, anti-anamorphosis involves filtering and interpolation processes that modify the information conveyed by the image. Moreover, to ensure accurate calculations for all points on an omnidirectional image, local anti-anamorphosis should be performed around each of these pixels, which considerably complicates calculation of the process and hampers uniform processing of the entire original image.

Daniilidis et al. [3] developed one of the first approaches to tailor filtering processes to omnidirectional images. Their proposal was - rather than working in an omnidirectional image space - to project the image plane in a space where the information is not very distorted relative to the real scene. They naturally opted to exemplify their approach by using the dome implemented in Geyer's model (Fig. 9). The proposed projection did not in any way alter the image information as the illumination values were not interpolated or filtered. The cost of this choice is obviously that the projected points are not regularly distributed over the sphere. The authors then defined kernels in the spherical coordinate space and proposed to perform convolution on the sphere surface. This technique can be used to estimate optical flow in an omnidirectional image sequence. It involves convolution operations that are highly complex because the values upon which the operations are based are not regularly distributed over the sphere whereas the kernels have a regular distribution. Apart from the calculation complexity, the problem with this type of method is that it disregards the Cartesian sampled nature of the initial image.
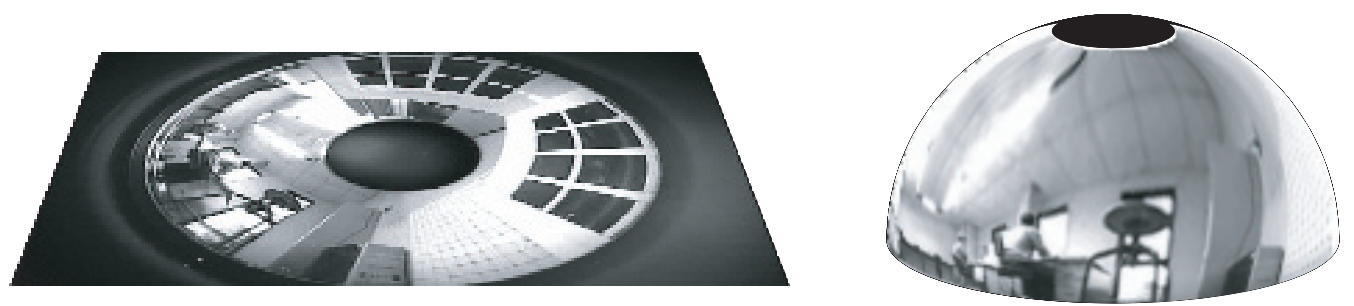

Figure 9: Projection of the Daniilidis dome.

Ieng et al. [4] proposed another use of the projective aspect of anamorphosed images. Their studies focused on mapping indices derived from two consecutive omnidirectional video sequence images. By a standard approach, a neighborhood can be defined when the variation domain of position parameters for objects relative to the sensor is known, and this neighborhood can subsequently be used as a basis for similarity calculations. This reasoning cannot apply for omnidirectional images since the same displacement amplitude will not have the same effect on an object depending on the position of its projection on the image. These authors proposed to solve this problem by redefining the neighborhood of each pixel by projecting on the image a kernel defined in a 3D space at a given distance from the sensor. They overcame the problem of determining the distance between the object and the sensor by using nested neighborhoods corresponding to decreasing distances between these two elements. Apart from the fact that the projective space used is not the equivalent sphere but rather its mirror image, this method differs from the previous one by the fact that the calculation is done directly on the anamorphosed image. Here again, however, the sampled aspect of the image is disregarded.

\section{Morphological operations on omnidirectional images}

Here we propose a variable structuring element based method using features of single viewpoint omnidirectional lenses. We use Geyer's model, as in the approach of Daniilidis, to determine relations between the 3D space and the projected space. Like Ieng, we project structuring elements on an anamorphosed image. Fuzzy structuring element are used to account for the nested aspect of binary structuring elements and Cartesian sampling of omnidirectional images. Our aim was to develop morphological operations that have the same properties for omnidirectional images as those devoted to standard projective images - a shape projected at a constant distance from the sensor in an omnidirectional image should be processed in the same 
way regardless of its position relative to the sensor. This property is, however, hampered by the image sampling. To take the discretization produced by the Cartesian retina into account, an image pixel is considered as a restriction of the space defined by the image plane whose illumination value is unknown.

\subsection{Morphological operation with a variable structuring element}

We feel, like all authors who have dealt with similar topics, that the projective space used should be chosen according to the application. Both the mirror space used by Ieng and the equivalent dome space of Daniilidis seem to be very suitable for dealing with this problem. We consider, however, that it is important to use a projective method that involves simple calculations, which was the basis of our choice of Geyer's method. We use a surrounding cylinder as projective space to illustrate our variable structuring element based projective morphology approach (Fig. 8).

As noted earlier, the surface occupied by a structuring element relative to the pixel size is linked (for a given calibration) with the presumed distance between the object and the sensor. This distance is represented by the diameter of the projection cylinder in the projective model that we implement (Fig. 10). This approach to the structuring element size is the same as that proposed by Ieng (for kernels), whereas Daniilidis represents it by the radius of an equivalent sphere. Nested structuring elements of decreasing sizes can be associated with increasing cylinder diameter values (Fig. 11).

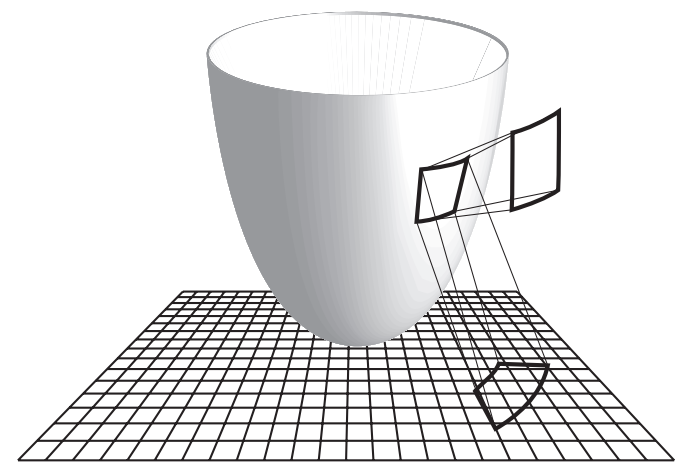

Figure 10: Structuring element projection.

A minimal diameter can be defined for the cylinder, e.g. the diameter of a physical cylinder surrounding a mirror. The family of nested structuring elements would then be supported by the structuring element defined by the minimal diameter, with the mode being a structuring element, i.e. a point defined by an infinite diameter. As the distance to the object is unknown, the probabilistic approach would involve mapping a probability distribution to the distance space while deducing a binary structuring element as a good tradeoff. Irrespective of the probability distribution selected for the distances, the confidence measure that can be mapped to each structuring element increases as the distance declines (Fig. 11). A linear fuzzy subset (i.e. a fuzzy subset with a linear membership function) whose support is defined by the minimal diameter and mode is defined by the infinite diameter will specifically represent a set of nested structuring elements independently of the selected probability distribution [12].
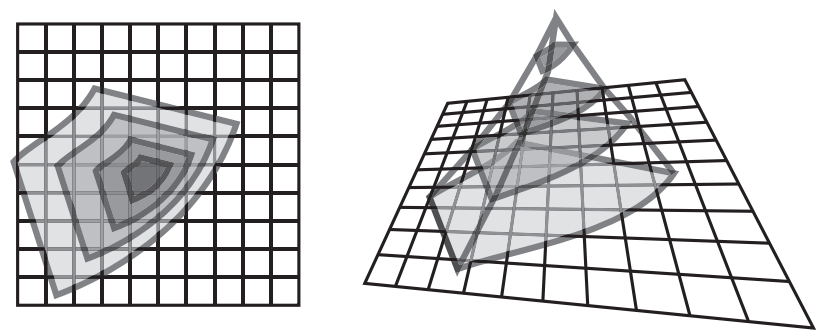

Figure 11: Nested structuring elements and a fuzzy structuring element.

\subsection{Taking sampling into account}

Figure 10 shows the projection of a binary structuring element onto a cylinder of a given radius. This kind of projected structuring element cannot be represented by a set of whole pixels. The least specific solution would be to use its surrounding envelope as a discrete structuring element representation (Fig. 12.a). As a more detailed model, the compatibility between the projected structuring element and each image pixel should be measured to obtain a discrete representation (Fig. 12.b). 

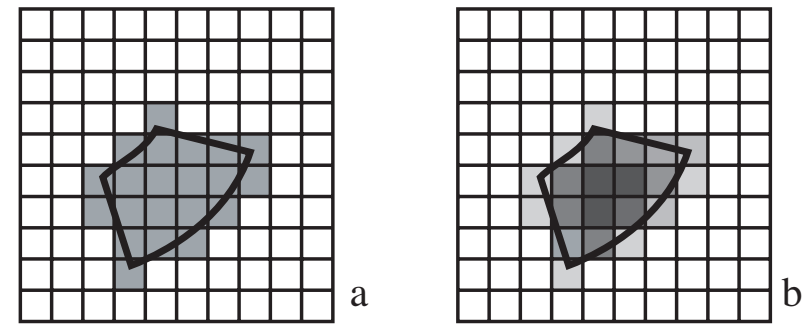

Figure 12: Interaction between a projected structuring element and image tiling.

The standard sampling representation model uses summative kernels to map an image plane probability domain to each sampled illumination measurement (Fig. 13.a). This probability domain can indiscriminately represent integration phenomena that give rise to an illumination measurement, a spatial domain in which the illumination measurement can be considered as ergodic or a domain within which this illumination value can be taken into account to interpolate the illumination. This gives a measurement of the compatibility between a pixel and a spatial domain by integrating a summative kernel on the structuring element. However, the choice of probability distribution has a marked impact on the results, as confirmed in all studies that focused on estimating illumination gradients [13].
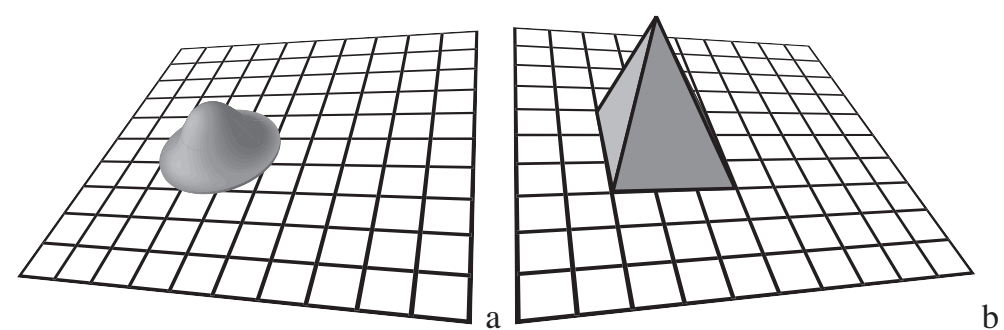

Figure 13: A summative kernel (a) and a fuzzy kernel (b).

The compatibility can also be determined by seeking the highest probability value whose associated confidence interval intersects the domain to be discretized. This possibilistic interpretation gives a higher compatibility measurement - this is a non-summative representation [14].

When considering the illumination localization imprecision induced by sampling, the mode of all monomodal summative kernels will be the pixel center, with the support being the domain encompassing its eight neighbors (Fig. 13.b). A linear (pyramidal) fuzzy subset that has this support and mode generalizes all confidence intervals that can be generated from this kernel class [12]. The upper compatibility measurement is then given by the possibility measurement of the structuring element restricted to the pixel (Fig. 14), while the lower compatibility measurement is obtained by complementation [15].

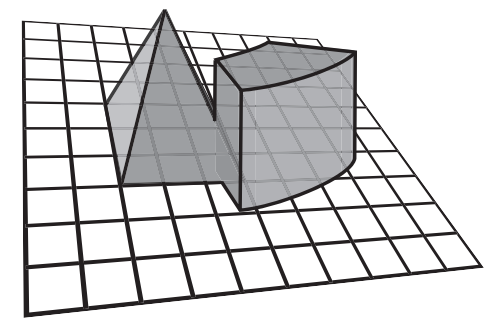

Figure 14: The upper compatibility.

\subsection{Interaction matrix.}

\subsubsection{Definition of the interaction matrix.}

The fuzzy neighborhood projected by each pixel of an omnidirectional image must be determined so as to be able to perform variable structuring element based morphological operations. The mode of this neighborhood is given by the position of the pixel whereas its geometry is defined in the most suitable projective space (here the cylinder of the smallest diameter). The fuzzy morphology that we attempt to define uses an interaction between this fuzzy neighborhood and that representing the Cartesian image sampling.

To simplify the notation, we will consider an image as a set of $\mathrm{N}$ pixels $\mathrm{P}_{\mathrm{n}}$ whose grey level is defined by $I_{n}$ (with $n$ ranging from 1 to $N$ ) and whose image coordinates are $\left(u_{n}, v_{n}\right)$. The image is considered as a fuzzy 
partition of the $\mathbb{R}^{2}$ plane, with a pyramidal fuzzy subset of $\mathbb{R}^{2}$ being mapped to each pixel $\mathrm{P}_{\mathrm{n}}$.

A neighborhood $\mathrm{V}\left(\mathrm{P}_{\mathrm{k}}\right)$ can be mapped to each pixel $\mathrm{P}_{\mathrm{k}}$ by using the extension principle applied in equation (4). Interactions $\beta_{k, n}$ between image pixel $P_{n}$ and the omnidirectional neighborhood $V\left(P_{k}\right)$ of pixel $P_{k}$ can be defined by the possibility of $\mathrm{P}_{\mathrm{n}}$ restricted to $\mathrm{V}\left(\mathrm{P}_{\mathrm{k}}\right)$ :

$\beta_{\mathrm{k}, \mathrm{n}}=\Pi\left(\mathrm{V}\left(\mathrm{P}_{\mathrm{k}}\right) ; \mathrm{P}_{\mathrm{n}}\right)=\operatorname{Sup}_{(\mathrm{u}, \mathrm{v}) \in \mathbb{R}^{2}}\left\{\min \left(\mu_{\mathrm{V}\left(\mathrm{P}_{\mathrm{k}}\right)}(\mathrm{u}, \mathrm{v}), \mu_{\mathrm{P}_{\mathrm{n}}}(\mathrm{u}, \mathrm{v})\right)\right\}$.

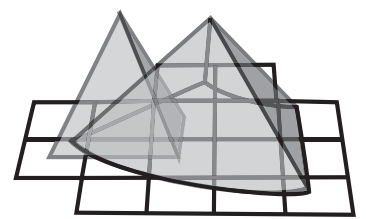

Figure 15: Interaction between a pixel and a neighborhood.

This interaction measurement represents the possibility of a fuzzy set of $\mathbb{R}^{2}$ elements belonging to both $\mathrm{P}_{n}$ and $\mathrm{V}\left(\mathrm{P}_{\mathrm{k}}\right)$. It can be considered as the highest $\alpha$ value that enables an intersection between $\mathrm{V}\left(\mathrm{P}_{\mathrm{k}}\right)$ and $\mathrm{P}_{\mathrm{n}}$ alpha cuts (Fig. 15), and can be calculated on the basis of this principle (see Appendix).

For our purposes, an interaction matrix is a (hollow) matrix $\mathrm{NxN}$ of $\beta_{\mathrm{k}, \mathrm{n}}$. This matrix is used to redefine basic morphological operations. The $\beta_{\mathrm{k}, \mathrm{n}}$ depend only on the sensor, so they can be precalculated. By construction $\beta_{\mathrm{k}, \mathrm{k}}=1$.

\subsubsection{Simplified interaction calculation.}

The membership function of $\mathrm{P}_{\mathrm{n}}$ is a $2 \mathrm{D}$ pyramidal fuzzy set, i.e. a Cartesian product of two fuzzy numbers $\mathrm{U}_{\mathrm{n}}$ and $\mathrm{V}_{\mathrm{n}}: \mathrm{P}_{\mathrm{n}}=\mathrm{U}_{\mathrm{n}} \times \mathrm{V}_{\mathrm{n}}$. The mode of $\mathrm{U}_{\mathrm{n}}\left(\mathrm{rsp} . \mathrm{V}_{\mathrm{n}}\right)$ is $\mathrm{u}_{\mathrm{n}}\left(\mathrm{rsp} . \mathrm{v}_{\mathrm{n}}\right)$ and its spread is defined by the image sampling interval $\partial \mathrm{u}$ (rsp. $\partial \mathrm{v})$ (Fig. 16).

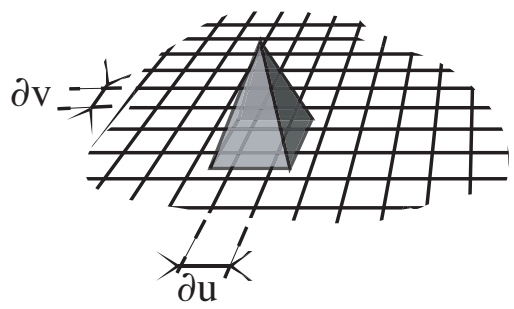

Figure 16: A fuzzy pixel.

Direct application of the extension principle to assess $\beta_{\mathrm{k}, \mathrm{n}}$ interactions could be very complicated. The properties of fuzzy numbers LR [16] are often used to determine $\mathrm{V}\left(\mathrm{P}_{\mathrm{k}}\right)$, i.e. the neighborhood projected in each pixel $\mathrm{P}_{\mathrm{k}}$. Variations in $\theta$ are the same in the image space and the cylinder space, but variations in $\mathrm{d}$ are nonlinear and defined by:

$\Delta d=\frac{d^{2}}{\varphi+\xi}\left|1-\frac{\xi^{2} \rho d}{\varphi+\xi+\rho}\right| \Delta \rho$,

with $\rho$ defined by equation (5) and $d=\sqrt{\left(\frac{\mathrm{u}-\mathrm{u}_{0}}{\mathrm{k}_{\mathrm{u}}}\right)^{2}+\left(\frac{\mathrm{v}-\mathrm{v}_{0}}{\mathrm{k}_{\mathrm{v}}}\right)^{2}}$.

The support of $\mathrm{V}\left(\mathrm{P}_{\mathrm{k}}\right)$ is defined by the polygon obtained for calculated $\Delta \mathrm{d}$ and $\Delta \theta$ values (Fig. 15). In this case, $\beta_{\mathrm{k}, \mathrm{n}}$ can be simply estimated through a dichotomous search for the highest alpha value such that alpha cuts in $\mathrm{V}\left(\mathrm{P}_{\mathrm{k}}\right)$ and $\mathrm{P}_{\mathrm{n}}$ have a non-null intersection. This representation can be simplified by just using the projection of terminal points of the support (Fig. 17.a) or by using the projection envelope of this polygon on axes $\mathrm{u}$ and $\mathrm{v}$ (Fig. 17.b). The restriction possibility is then defined by conjunction of the restricted possibilities of these projections on axes $u$ and $v . \beta_{k, n}$ is always overestimated in the latter case (see Appendix for algorithms).

Each simplification of the $\beta_{k, n}$ calculation reduces the specificity of the thus determined neighborhood $\mathrm{V}\left(\mathrm{P}_{\mathrm{k}}\right)$ but increases the interaction matrix calculation rate. Morphologically processed images will be even more fuzzy because the neighborhoods are less specific. 

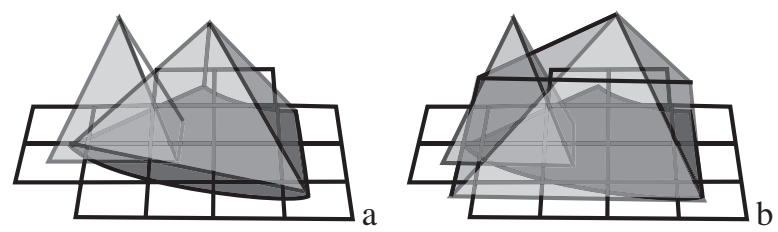

Figure 17: Simplifications of $\mathrm{V}\left(\mathrm{P}_{\mathrm{k}}\right)$.

\subsubsection{Discussion on the interaction matrix}

Obviously there could be other possible methods for calculating the interaction matrix than the one we propose here. The neighborhoods that we suggest are based on a definition of balls using the Manhattan metric. We feel that this approach best complies with the Cartesian aspect of digital image representation. The simplicity of calculations that we obtain also seem to confirm the validity of this technical choice. However, kernels based on isotropic distances, e.g. Euclidian distance, are often preferred by the image processing community. This distance can be used without any loss of generality for the variable structuring element based morphological methods that we propose. The only drawback is that the interaction calculations are much more time consuming.

Standard summative kernels could also be used to calculate the interaction matrix. This approach involves calculating the value of an interaction between pixel $P_{n}$ and neighborhood $V\left(P_{k}\right)$ by integration of a summative kernel that defines the projected neighborhood $\mathrm{V}\left(\mathrm{P}_{\mathrm{k}}\right)$ with a summative kernel defined by $\mathrm{P}_{\mathrm{n}}$. For uniform kernels, this simply involves an area ratio calculation. This solution, as illustrated in Figure 18, is very time consuming for computation. For monomodal symmetrical kernels, the value of interaction $\beta_{k}, n$ can be calculated from the distance that separates modes of the two kernels, respectively representing $V\left(P_{k}\right)$ and $\mathrm{P}_{\mathrm{n}}$.

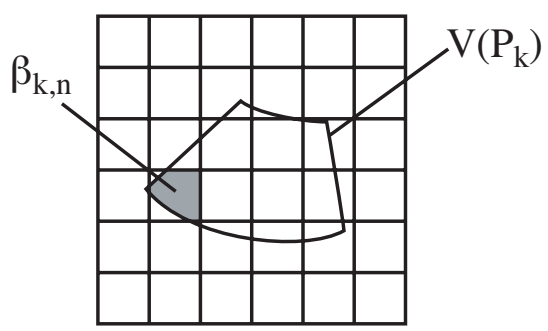

Figure 18: Probabilistic interaction using a uniform kernel.

When the neighborhood is defined by a summative kernel, it is essential to normalize the possibility distributions obtained in compliance with property (8):

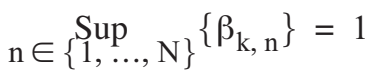

This normalization can be done by dividing each coefficient $\beta_{k, n}$ by $n_{f}\{1, \ldots, N\}$
probability/possibility transformation described by Dubois and Prade $\left\{\beta_{k, n}\right\}$, or by using the

\subsection{Dilation extension}

\subsubsection{Extension via the Choquet integral}

What is dilation? Rigorously, a dilation is an operation defined on a lattice that commutes with the supremum of the lattice. In the particular case where the underlying space is affine, specific dilations can be defined, based on a structuring element. From a fuzzy morphology standpoint, the value of a dilated image $\mathrm{D}\left(\mathrm{I}_{\mathrm{k}}\right)$ at point $\mathrm{P}_{\mathrm{k}}$ is the maximum value of the grey level distribution in the domain defined by the structuring element around $\mathrm{P}_{\mathrm{k}}$. In terms of image transformation, a dilation fills the valleys and flattens the peaks - it homogenizes and clarifies the image and tends to erase dark objects around the same size as the structuring element, while spacing out the pale objects

In the case we are investigating here, the neighborhood $\mathrm{V}\left(\mathrm{P}_{\mathrm{k}}\right)$ is not defined in a binary way but rather by its $\beta_{k, n}$ interactions with all $P_{n}$ image pixels. A better estimation of the grey level of $V\left(P_{k}\right)$ can be obtained, when the grey levels of $P_{n}$ and interactions are known, by using the Choquet integral [14, 17], in the following form: 
$\mathrm{D}_{\mathrm{c}}\left(\mathrm{I}_{\mathrm{k}}\right)=\sum_{\mathrm{n}=1}^{\mathrm{N}} \mathrm{I}_{(\mathrm{n})}\left(\mathrm{v}\left(\mathrm{A}_{(\mathrm{n})}\right)-\mathrm{v}\left(\mathrm{A}_{(\mathrm{n}+1)}\right)\right)$,

the index notations (.) indicate a permutation that sorts the image grey levels such that $\mathrm{I}_{(1)} \leq \mathrm{I}_{(2)} \leq \ldots \leq \mathrm{I}_{(\mathrm{N})}$. The sets $\mathrm{A}_{(\mathrm{n})}=\left\{\mathrm{P}_{(\mathrm{n})}, \ldots, \mathrm{P}_{(\mathrm{N})}\right\}$ are binary coalitions of $\mathbb{R}^{2}$ that pool all pixels whose grey level is higher or equal to $\mathrm{I}_{(\mathrm{n})} \cdot \mathrm{v}\left(\mathrm{A}_{(\mathrm{n})}\right)$ is a confidence measure ${ }^{1}$ that can be assigned to set $\mathrm{A}_{(\mathrm{n})}$ to determine the grey level value of the dilated image to point $P_{k}$ with a hypothetical grey level threshold of $I_{(n)}$.

Coalition $\mathrm{A}_{(\mathrm{n})}$ could be considered as a discrete fuzzy set of fuzzy pixels with the same incongruous confidence levels (Fig. 19).

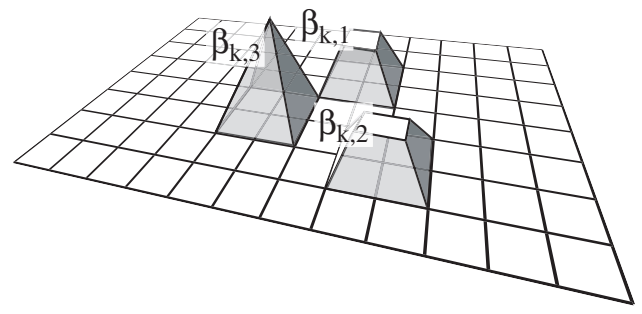

Figure 19: Coalition of three pixels.

The largest possible confidence value in this coalition is given by:

$\mathrm{v}\left(\mathrm{A}_{(\mathrm{n})}\right)=\operatorname{Sup}_{\mathrm{i} \in\{\mathrm{n}, \ldots, \mathrm{N}\}}\left\{\beta_{\mathrm{k},(\mathrm{i})}\right\}$

The confidence measure distribution is normalized since $\beta_{\mathrm{k}, \mathrm{k}}=1$ and thus $\mathrm{v}\left(\mathrm{A}_{(0)}\right)=1$. Using the Choquet interval and the confidence measure $\mathrm{v}\left(\mathrm{A}_{(\mathrm{n})}\right)$ enables us to determine a morphological expansion operator whose definition is compatible with the first dilation extension proposal of Isabelle Bloch [2]. She demonstrated that this generalization gives an operator that is not compatible with union. This dilation extension therefore cannot be called dilation. It has been shown, however, that dilation of the union of two images is always greater than the dilation union of the same images:

$\mathrm{D}_{\mathrm{c}}\left(\max \left(\mathrm{I} 1_{\mathrm{k}}, \mathrm{I} 2_{\mathrm{k}}\right)\right) \geq \max \left(\mathrm{D}_{\mathrm{c}}\left(\mathrm{I} 1_{\mathrm{k}}\right), \mathrm{D}_{\mathrm{c}}\left(\mathrm{I} 2_{\mathrm{k}}\right)\right)$.

The proposed dilation extension is thus the upper boundary for dilation operators.

\subsubsection{Extension via the Sugeno integral.}

An extension based on the Sugeno integral should preferably be used when commutation with the union properties is to be maintained. This extension requires image fuzzification, i.e. the assignment of a membership value to each grey level must be meaningful. The easiest method involves mapping a linear membership function to the image grey level, while being careful to always use the same upper and lower threshold values for the image. For instance, with 8-bit coded images, a grey level value of 255 can represent full membership, with a lack of membership being 0 (Fig. 20).

The original classification can be reallocated as follows:

$\mathrm{D}_{\mathrm{s}}\left(\mathrm{I}_{\mathrm{k}}\right)=\mathrm{n} \in\{1, \ldots, \mathrm{N}\}_{\operatorname{Sup}}\left\{\min \left(\mu\left(\mathrm{I}_{(\mathrm{n})}\right), \mathrm{v}\left(\mathrm{A}_{\mathrm{n}}\right)\right)\right\}$,

the index notations (.) indicate a permutation that sorts the image grey levels such that $\mathrm{I}_{(1)} \leq \mathrm{I}_{(2)} \leq \ldots \leq \mathrm{I}_{(\mathrm{N})}$. Each set $\mathrm{A}_{(\mathrm{n})}$ and its associated confidence measure are determined in the same way as for the Choquet integral. While taking the confidence measure defined by (10) into account, equation (12) can be simplified as:

$\mathrm{D}_{\mathrm{s}}\left(\mathrm{I}_{\mathrm{k}}\right)=\operatorname{\mathrm {nup}}_{\mathrm{n} \in\{1, \ldots, \mathrm{N}\}}\left\{\min \left(\mu\left(\mathrm{I}_{\mathrm{n}}\right), \beta_{\mathrm{k}, \mathrm{n}}\right)\right\}$.

1. Historically, this value is called capacity. 


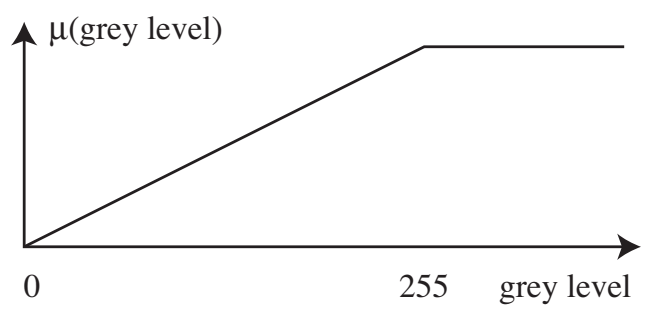

Figure 20: Mapping membership functions to grey levels

Hence, sorting is not required for generalization of the dilation by the Sugeno integral. The obtained operator meets all conditions required for it to be classified as a dilation.

\subsection{Erosion and morphological gradient extension}

From a functional morphology standpoint, the value of eroded image $\mathrm{E}\left(\mathrm{I}_{\mathrm{k}}\right)$ at point $\mathrm{P}_{\mathrm{k}}$ is the minimum greylevel distribution value in the domain defined by the structuring element around $\mathrm{P}_{\mathrm{k}}$. From an image transformation standpoint, erosion broadens the valleys and flattens the peaks. This process homogenizes and darkens the image, while tending to erase pale objects about the same size as the structuring element and to expand dark objects.

The easiest way to halt erosion is to utilize the complementarity of morphological operations. For extension using the Choquet interval, the $\mathrm{E}_{\mathrm{c}}\left(\mathrm{I}_{\mathrm{k}}\right)$ value represents the grey level of pixel $\mathrm{P}_{\mathrm{k}}$ after erosion:

$\mathrm{E}_{\mathrm{c}}\left(\mathrm{I}_{\mathrm{k}}\right)=-\sum_{\mathrm{n}=1}^{\mathrm{N}}-\mathrm{I}_{(\mathrm{n})}\left(\mathrm{v}\left(\mathrm{A}_{(\mathrm{n})}\right)-\mathrm{v}\left(\mathrm{A}_{(\mathrm{n}+1)}\right)\right)$,

where (.) is a permutation that sorts the image grey levels such that $-\mathrm{I}_{(1)} \leq-\mathrm{I}_{(2)} \leq \ldots \leq-\mathrm{I}_{(\mathrm{N})}$. $\mathrm{A}_{(\mathrm{n})}$ coalitions and associated capacities are determined in the same way. Note that Equation (14) is exactly the same as Equation (9) when sorting the $I_{n}$ in the reverse order. This property can be used to speed up the computation when both erosion and dilation of an image are needed (only one sort is needed, e.g. for a morphological gradient computation).

For extension by the Sugeno integral, the complementarity has an especially simple form:

$\mathrm{E}_{\mathrm{s}}\left(\mathrm{I}_{\mathrm{k}}\right)=\operatorname{Inf}_{\mathrm{n} \in\{1, \ldots, \mathrm{N}\}}\left\{\max \left(\mu\left(\mathrm{I}_{\mathrm{n}}\right),\left(1-\beta_{\mathrm{k}, \mathrm{n}}\right)\right)\right\}$

We kept the standard definitions for gradient and filtering operations (opening and closing). Opening is defined by $\mathrm{O}\left(\mathrm{I}_{\mathrm{k}}\right)=\mathrm{D}\left(\mathrm{E}\left(\mathrm{I}_{\mathrm{k}}\right)\right)$, while closing is $\mathrm{C}\left(\mathrm{I}_{\mathrm{k}}\right)=\mathrm{E}\left(\mathrm{D}\left(\mathrm{I}_{\mathrm{k}}\right)\right)$. Finally we used a additive definition of gradient: $G\left(I_{k}\right)=D\left(I_{k}\right)-E\left(I_{k}\right)$. Note that the permutation that enables erosion determination is the inverse of that which defines the dilation. Only one sorting is thus required to compute the morphological gradient. It could, as suggested by Maragos et al. [18], also be interesting to test non-additive approaches.

\subsection{Discussion on local recognition when using the Choquet integral}

We defined operators suitable for single viewpoint omnidirectional images in order to determine the translation invariance and compatibility on the basis of bandwidth variations in the projected space. A morphological operator must, amongst different factors, comply with the local recognition property — which would seem to go against the dilation generalization obtained by the Choquet integral since the grey level of the entire image is involved in equation (9). This poor property seems to be a corollary to the lack of grey-level normalization. We shall try to demonstrate that the Choquet generalization respects the local recognition, i.e. there is a finite number of associated p pixels, such that:

$\mathrm{D}_{\mathrm{c}}\left(\mathrm{I}_{\mathrm{k}}\right)=\sum_{\mathrm{n}=1}^{\mathrm{p}} \mathrm{v}\left(\mathrm{A}_{(\mathrm{n})}\right)\left(\mathrm{I}_{(\mathrm{n})}-\mathrm{I}_{(\mathrm{n}-1)}\right)$.

These $\mathrm{p}$ pixels are all those such that $\beta_{\mathrm{k}, \mathrm{n}} \neq 0$. Let $\mathrm{I}_{(0)}=\mathrm{I}_{\min }$.

Equation (9) can also be written in the following form: 
$\mathrm{D}_{\mathrm{c}}\left(\mathrm{I}_{\mathrm{k}}\right)=\sum_{\mathrm{n}=\mathrm{N}}^{1}\left[_{\mathrm{i} \in\{\mathrm{nup}, \ldots, N\}}\left\{\beta_{\mathrm{k},(\mathrm{i})}\right\}\right]\left(\mathrm{I}_{(\mathrm{n})}-\mathrm{I}_{(\mathrm{n}-1)}\right)$.

Let $r$ be the first index from $N$ such that $\beta_{k,(r)} \neq 0$, so all the $v\left(A_{(n)}\right)$ from $N$ to $r$ are null. (17) is then rewritten as:

$\mathrm{D}_{\mathrm{c}}\left(\mathrm{I}_{\mathrm{k}}\right)=\mathrm{v}\left(\mathrm{A}_{(\mathrm{r})}\right)\left(\mathrm{I}_{(\mathrm{r})}-\mathrm{I}_{(\mathrm{r}-1)}\right)+\mathrm{v}\left(\mathrm{A}_{(\mathrm{r}-1)}\right)\left(\mathrm{I}_{(\mathrm{r}-1)}-\mathrm{I}_{(\mathrm{r}-2)}\right)+\ldots$

Either $\beta_{\mathrm{k},(\mathrm{r}-1)} \neq 0$, and therefore Pr-1 is a local neighborhood element, or $\beta_{\mathrm{k},(\mathrm{r}-1)}=0$ and then (18) becomes:

$\mathrm{D}_{\mathrm{c}}\left(\mathrm{I}_{\mathrm{k}}\right)=\mathrm{v}\left(\mathrm{A}_{(\mathrm{r})}\right)\left(\mathrm{I}_{(\mathrm{r})}-\mathrm{I}_{(\mathrm{s})}\right)+\mathrm{v}\left(\mathrm{A}_{(\mathrm{s})}\right)\left(\mathrm{I}_{(\mathrm{s})}-\mathrm{I}_{(\mathrm{s}-1)}\right)+\ldots$

Where $\mathrm{s}$ is the index following $\mathrm{r}$ such that $\beta_{\mathrm{k}, \mathrm{s}} \neq 0$. By the same reasoning, equation (19) involves only local information.

Finally, let $\mathrm{t}$ be the highest index such that $\beta_{\mathrm{k},(\mathrm{t})}=\underset{\mathrm{i} \in\{1, \ldots, \mathrm{N}\}}{\text { Sup }}\left\{\beta_{\mathrm{k},(\mathrm{i})}\right\}$, and the development of (19)
shows that:

$$
\mathrm{D}_{\mathrm{c}}\left(\mathrm{I}_{\mathrm{k}}\right)=\mathrm{v}\left(\mathrm{A}_{(\mathrm{r})}\right)\left(\mathrm{I}_{(\mathrm{r})}-\mathrm{I}_{(\mathrm{s})}\right)+\ldots+\mathrm{v}\left(\mathrm{A}_{(\mathrm{t})}\right)\left[\left(\mathrm{I}_{(\mathrm{t})}-\mathrm{I}_{(\mathrm{t}-1)}\right)+\ldots+\left(\mathrm{I}_{(1)}-\mathrm{I}_{(0)}\right)\right]
$$

with, by convention $\mathrm{I}_{(0)}=\mathrm{I}_{\min }$. Therefore:

$\mathrm{D}_{\mathrm{c}}\left(\mathrm{I}_{\mathrm{k}}\right)=\mathrm{v}\left(\mathrm{A}_{(\mathrm{r})}\right)\left(\mathrm{I}_{(\mathrm{r})}-\mathrm{I}_{(\mathrm{s})}\right)+\ldots+\mathrm{v}\left(\mathrm{A}_{(\mathrm{t})}\right)\left(\mathrm{I}_{(\mathrm{t})}-\mathrm{I}_{\min }\right)$.

This formula includes only the grey level of $\mathrm{P}_{\mathrm{n}}$ whose support intersects that of the neighborhood $\mathrm{V}\left(\mathrm{P}_{\mathrm{k}}\right)$ and $\mathrm{I}_{\min }$. Because of this property, the Choquet extension can be used with only one local sorting in the neighborhood of the analyzed pixel, thus markedly reducing the computation time.

\subsection{Morphological anti-anamorphosis}

All of the techniques that we have just presented can facilitate creation of a perspective image from a calibrated omnidirectional image. This so-called anti-anamorphosis process was presented in section 2.3. This operation is performed by defining the sampled projective space that is to undergo anti-anamorphosis (projection cylinder, limited plane, etc.) and then calculating the interaction matrix that is used to map a sampled fuzzy neighborhood $\mathrm{V}\left(\mathrm{P}_{\mathrm{k}}\right)$ of the anamorphosed image to each pixel $\mathrm{P}_{\mathrm{k}}$ of the sought-after image (Fig. 21). It should be noted that in this case the possibility distribution mapped to neighborhood $\mathrm{V}\left(\mathrm{P}_{\mathrm{k}}\right)$ is not normalized by construction, i.e. $\operatorname{Sup}_{\mathrm{n}} \operatorname{Sup}_{\{1, \ldots, \mathrm{N}\}}\left\{\beta_{\mathrm{k}, \mathrm{n}}\right\} \neq 1$.

Image anti-anamorphosis thus involves estimating the grey level of each projected image pixel via the known value of the set of anamorphosed omnidirectional image pixels. This estimation can be done in two different ways, i.e. using an additive or a non-additive method.

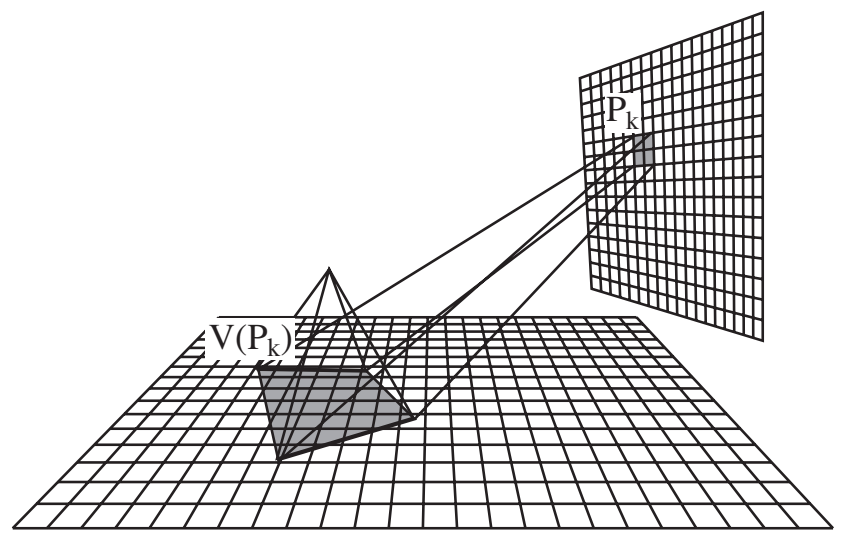

Figure 21: Calculation of the interaction neighborhood.

\subsubsection{Additive anti-anamorphosis}

The additive method considers each element $\beta_{\mathrm{k}, \mathrm{n}}$ of an interaction matrix as a confidence measure to be assigned to pixel $\mathrm{n}$ of an anamorphosed image to estimate the value of pixel $\mathrm{k}$ of anti-anamorphosed images. The $\mathrm{Id}_{\mathrm{k}}$ value of the anti-anamorphosed image grey level is then obtained by a barycentric-type additive 
clustering operation (22):

$\mathrm{Id}_{\mathrm{k}}=\left(\sum_{\mathrm{n}} \beta_{\mathrm{k}, \mathrm{n}} \mathrm{I}_{\mathrm{n}}\right) /\left(\sum_{\mathrm{n}} \beta_{\mathrm{k}, \mathrm{n}}\right)$

\subsubsection{Non-additive anti-anamorphosis.}

The non-additive method considers each element $\beta_{\mathrm{k}, \mathrm{n}}$ of an interaction matrix as a confidence measure to be assigned to pixel $\mathrm{n}$ of an anamorphosed image to classify the value of pixel $\mathrm{k}$ of anti-anamorphosed images. As the possibility distribution is not normalized, the $\beta_{\mathrm{k}, \mathrm{n}}$ distribution should be replaced by its normalized equivalent:

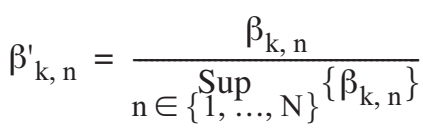

The $\mathrm{Id}_{\mathrm{k}}$ value for the grey level of an anti-anamorphosed image is obtained by a non-additive clustering operation analogous to those we outlined in section 3.4. Two anti-anamorphosed images can thus be produced that are respectively eroded and dilated versions of the initial anti-anamorphosed image. The $D\left(\operatorname{Id}_{k}\right)$ value of the dilated image in $\mathrm{P}_{\mathrm{k}}$ is the maximum possible grey-level distribution value in the domain defined by the projected structuring element $\mathrm{V}\left(\mathrm{P}_{\mathrm{k}}\right)$. The $\mathrm{E}\left(\mathrm{Id}_{\mathrm{k}}\right)$ value of the eroded image in $\mathrm{P}_{\mathrm{k}}$ is the minimum possible grey-level distribution value in the domain defined by the projected structuring element $\mathrm{V}\left(\mathrm{P}_{\mathrm{k}}\right)$. This antianamorphosis is imprecise since the grey-level value for each point is imprecisely known $\left[\mathrm{E}\left(\operatorname{Id}_{\mathrm{k}}\right), \mathrm{D}\left(\operatorname{Id}_{\mathrm{k}}\right)\right]$. An image of the morphological edges of an anti-anamorphosed image can be obtained by subtracting the eroded image from the dilated image.

To obtain a projective anti-anamorphosed image, the eroded image has to be dilated or the dilated image must be eroded or, even better, the mean of the two produced images can be used. The dynamics of the image obtained by this method are generally better than those obtained by the additive method as the contrasts are better portrayed.

\section{Experiments}

Here we present four examples to illustrate some properties of the omnidirectional morphological operators obtained. The results presented were based on the Choquet method. Analogous visual results were obtained with the Sugeno method involving fuzzy grey-level classification described in section 3.4.2.

The images used were taken with a digital camera alternatively fitted with two single viewpoint catadioptric omnidirectional lenses - one paraboloid and the other hyperboloid.

\subsection{Experiment 1: Morphological filtering}

Figure 22 shows an omnidirectional image acquired with a paraboloid mirror, one with a frame to calibrate the sensor. This frame can be viewed as a set of black blocks on a white background or a set of white lines on a black background. Small black dots are found at the intersection of the white lines. In this first experiment, we performed morphological filtering by closure to get rid of the small black dots while inducing very little deformation of the other projected shapes of the frame. The standard morphological approach was compared to the projected fuzzy structuring element based approach. A square structuring element was used in both cases. It was mapped to the image space by the standard approach and in the unfolded cylinder plane by our approach. The size of this structuring element was the smallest that would enable us to get rid of the black dots. Standard closure led to irregular distortion of the black squares of the frame (Fig. 23), while omnidirectional closure better respected the geometry of the squares (Fig. 24). 

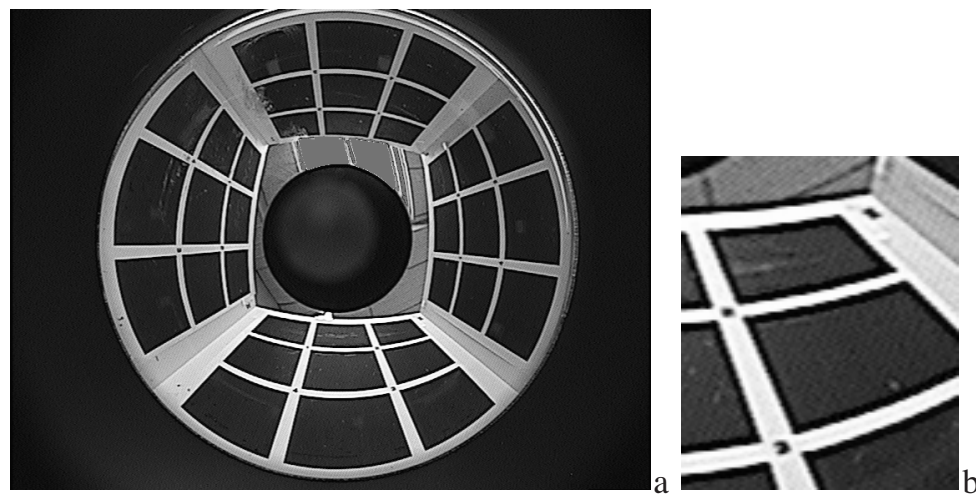

Figure 22: Original image and detailed view.
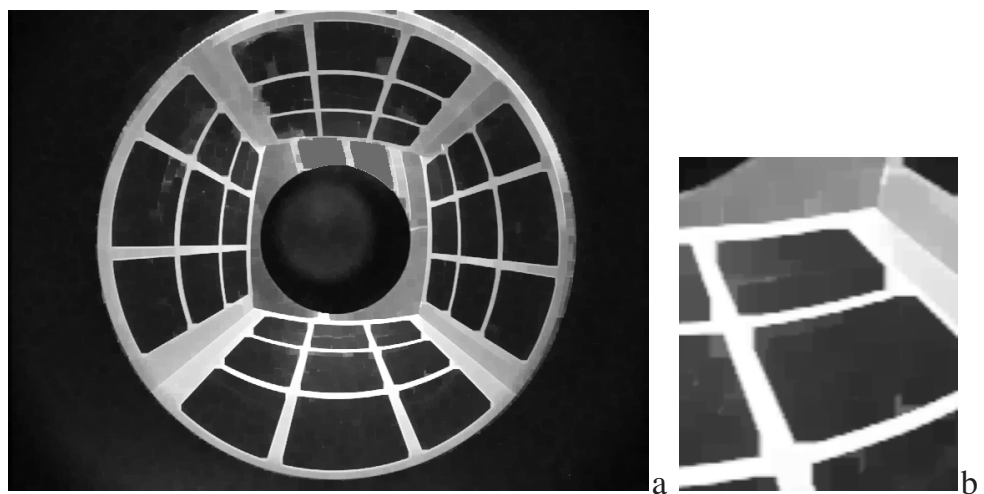

Figure 23: Standard closure and detailed view.
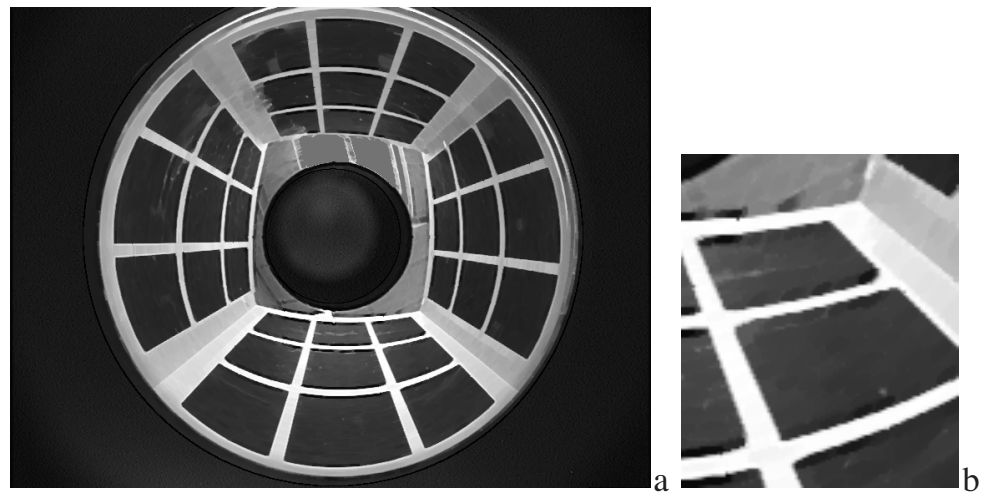

Figure 24: Omnidirectional closure and detailed view.

\subsection{Experiment 2: Oriented operation}

The second experiment highlights the suitability of the projected structuring element for oriented image processing operations. Here again we compare the omnidirectional approach to the standard approach, even though this comparison is less meaningful. Figure 25 shows an image of a chessboard taken with a camera fitted with a hyperboloid mirror along with two detailed views of the top and bottom of the chessboard, i.e. in two areas with different resolutions within the projected space. In this experiment, we wanted to induce an oriented dilation. Figure 26 shows a standard dilation with a 4-pixel wide structuring element oriented in the direction of the image lines. This shows that this operator is not valid for reducing the size of black squares projected in a particular direction (here the frame plane). It also indicates that reducing the size of the black squares via dilation does not process all of the projected squares in the same way throughout the projection area. This 4-pixel wide structuring element was projected on the cylinder around the edge of the mirror image. We thus determined the support of the projected fuzzy structuring element, which was tangential to the contour lines of the mirror. The results of this oriented dilation are shown in Figure 27. In the projected high-resolution zone, the morphological operator clearly performed as expected, i.e. it reduced the size of the squares in the direction of the structuring element (Fig. 27.b). Conversely, in the low-resolution zone, the projected structuring element is comparable to a fuzzy structuring element with respect to defining the pixel. The morphological operator performed like an operator whose structuring element is 
defined in the image space (Fig. 27.c). This highlights that the operators we propose take both the projective space and the sampled image space into account. Finally, the images presented in Figure 28 show an experiment similar to the directional dilation of the frame image (Fig. 22). Figure 28. a shows a dilation of the frame with a 25-pixel structuring element in the direction of the image lines, while Figure 28.b shows a dilation with a tangential structuring element projected from the previous structuring element. This latter experiment clearly shows the directional properties of the proposed morphological operators.
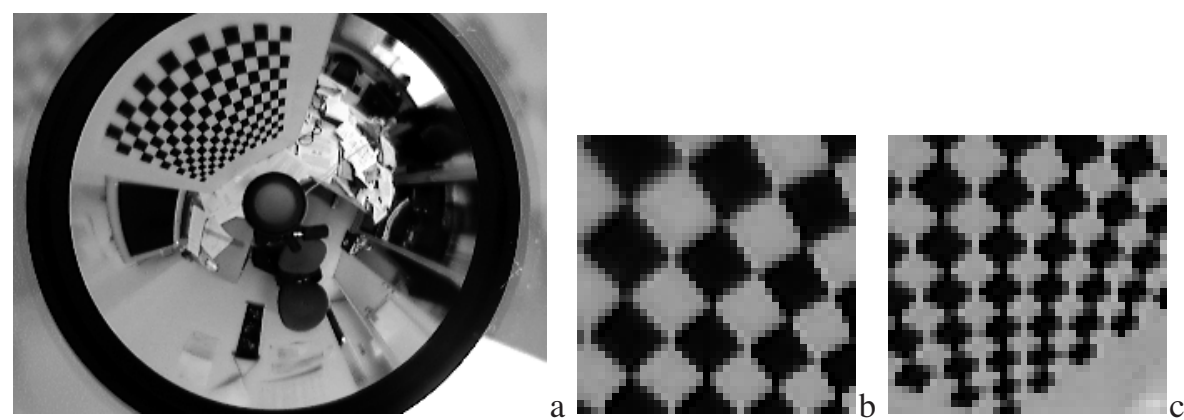

Figure 25: Hyperbolic image of a frame and detailed views.
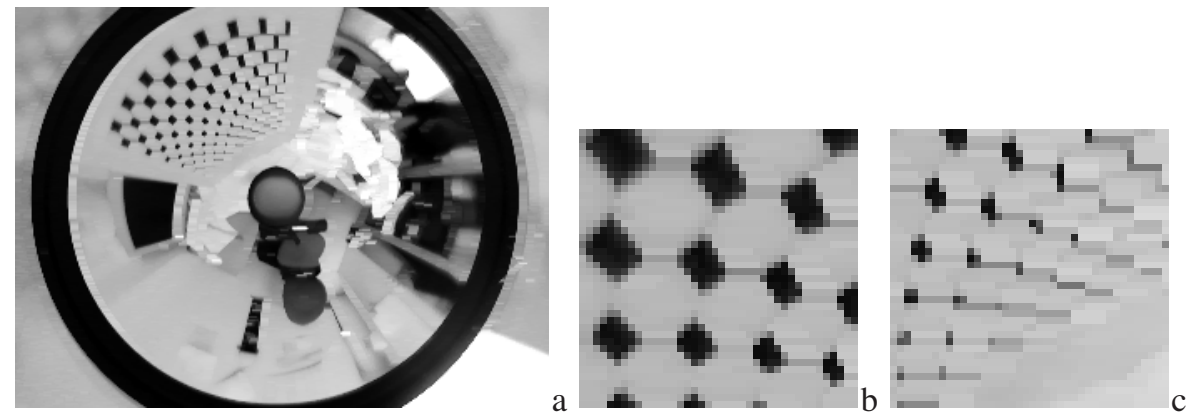

Figure 26: A standard dilation and detailed views.
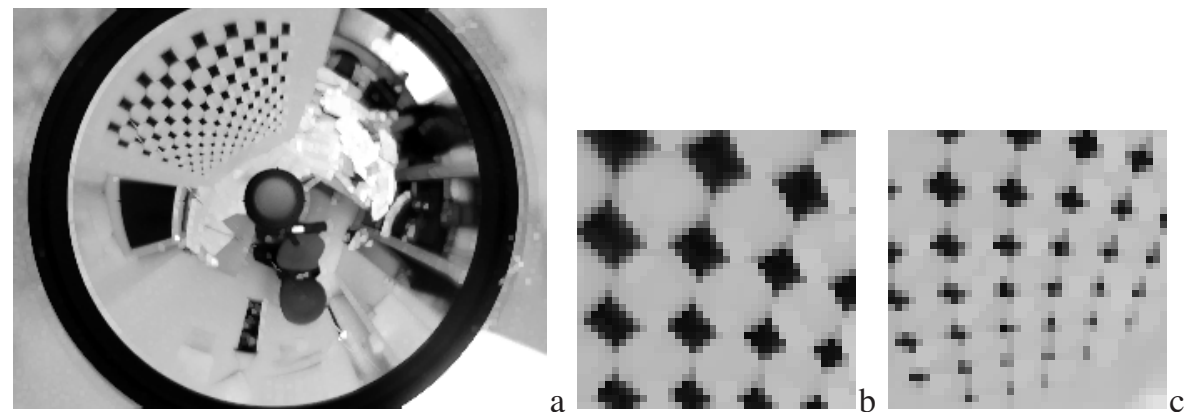

Figure 27: A tangential dilation and detailed views.
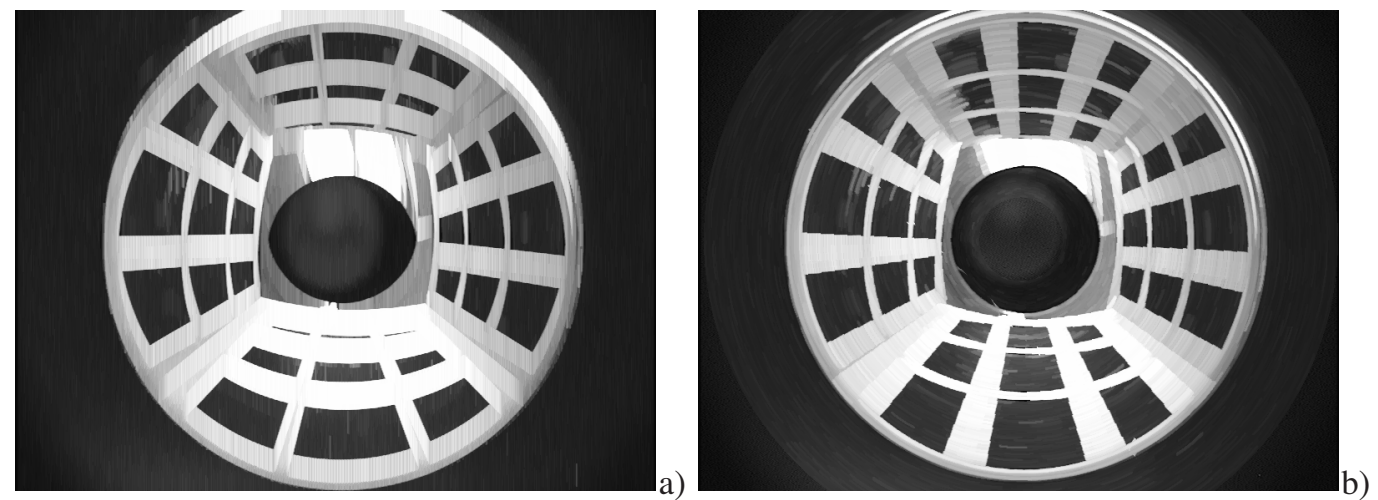

Figure 28: A directional dilation of the frame, a) standard dilation, b) tangential dilation.

\subsection{Experiment 3: Morphological gradient}

The third experiment was designed to extract the edges of the black squares from the parabolic image shown 
in Figure 22. Here we compared the projected structuring element based morphological approach with the standard morphological approach and also with the two additive approaches. In the four experiments, the structuring element was selected to be comparable to the projection of white lines around the edge of the mirror, which corresponds to a structuring element of $4 \times 4$ pixels for the standard morphological approach. This structuring element was projected on the cylinder when positioned at the edge of the mirror. The two morphological approaches clearly gave the same results in the first ring (mirror edge). However, only the edges obtained by using a projective structuring element were consistent with the projection of the edges of the black squares (Fig. 29, 30). We anti-anamorphosed the morphological gradient image in order to clearly demonstrate the projective aspect of the edges obtained. After anti-anamorphosis, the resulting edges were clearly regular in the projective space on the omnidirectional images (Fig. 34) but not on the standard images (Fig. 33).
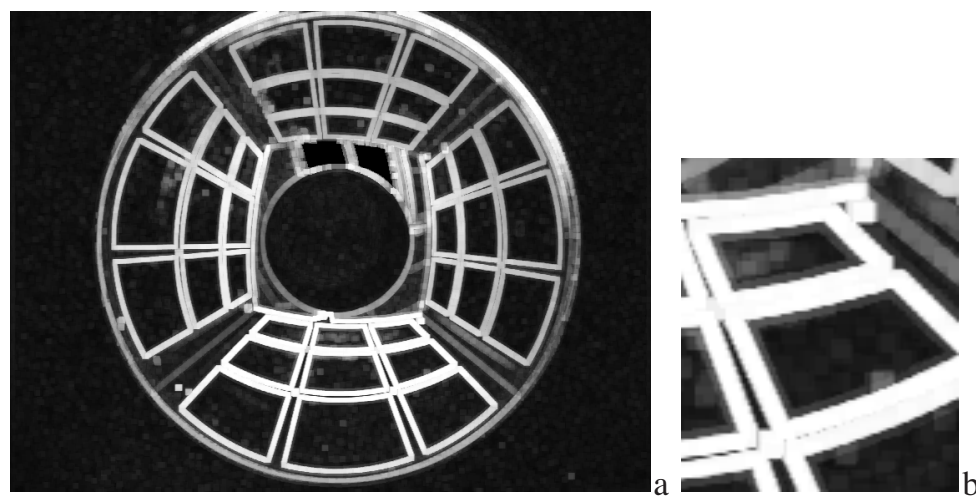

Figure 29: Standard gradient and detailed view.
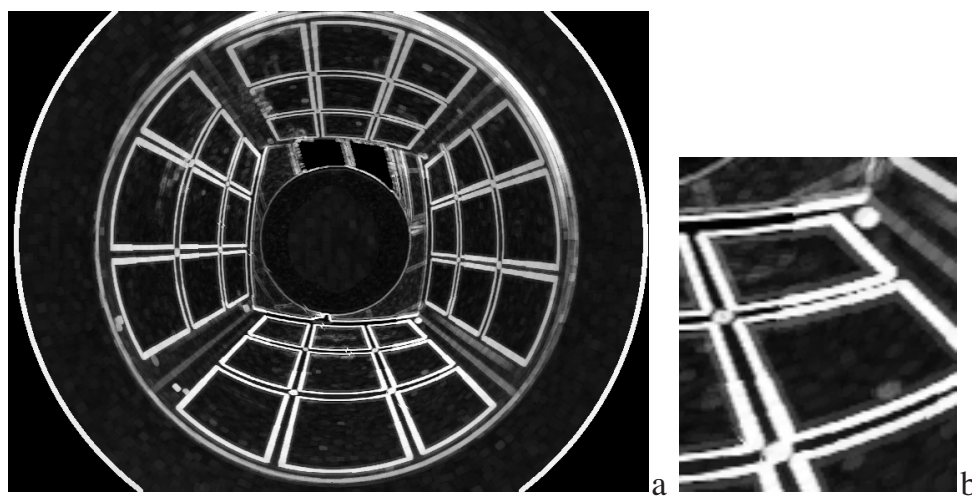

Figure 30: Omnidirectional gradient and detailed view.

We supplement the comparison with the standards of the gradients obtained by using the filters of Castan (Fig. 31) and Deriche (Fig. 32). The 90\% confidence interval of the summative kernels used had a support comparable to a $4 \times 4$ pixel box. The extracted images had a more subtle aspect. The filtering approaches and morphological approaches did not define the edges in the same way. However, it was noteworthy that the kernels selected here were too spread out to efficiently detect the corners. This detection was further hampered by the presence of small dots. The Castan filter poorly interpreted the corners (Fig. 31.b) whereas the Deriche filter detected rounded corners (Fig. 32.b). The edges of black dots at intersections of white lines were interpreted as noise by the Deriche filter and associated with the edges of black squares by the Castan filter. None of the morphological approaches had these defects. Finally, anti-anamorphosis of gradient images (Fig. 35, 36) highlighted the edges with variable sizes for the fixed summative kernel approach in the image space. Note that, for a fair comparison, a summative kernel of variable size should also be included. This problem has still not been solved. 


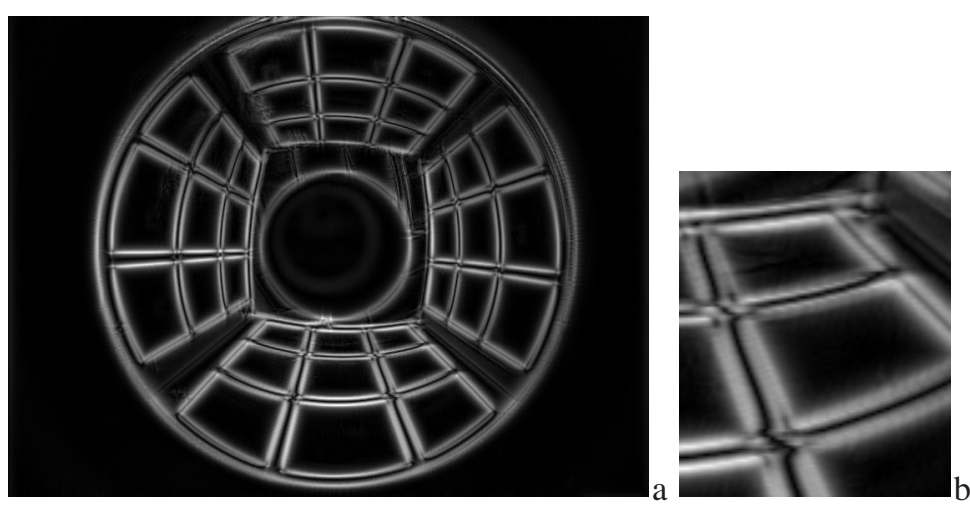

Figure 31: Castan gradient and detailed view.

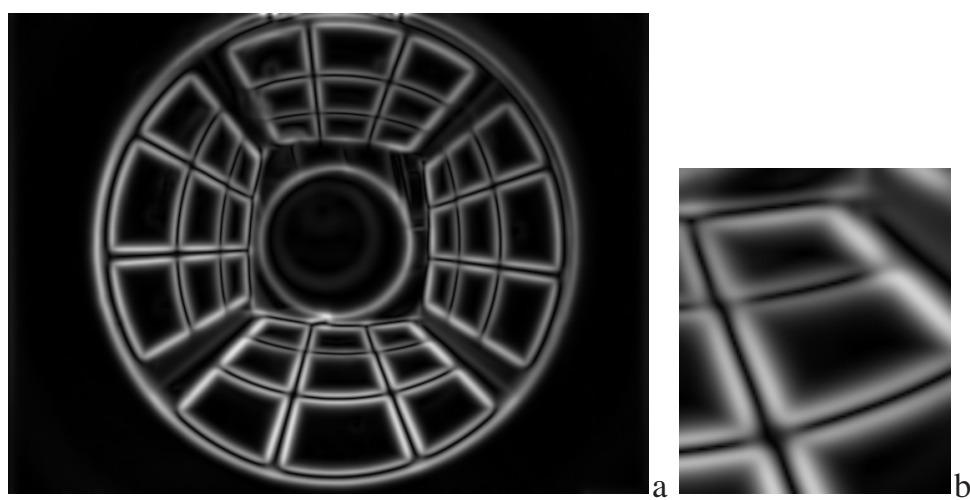

Figure 32: Deriche gradient and detailed view.

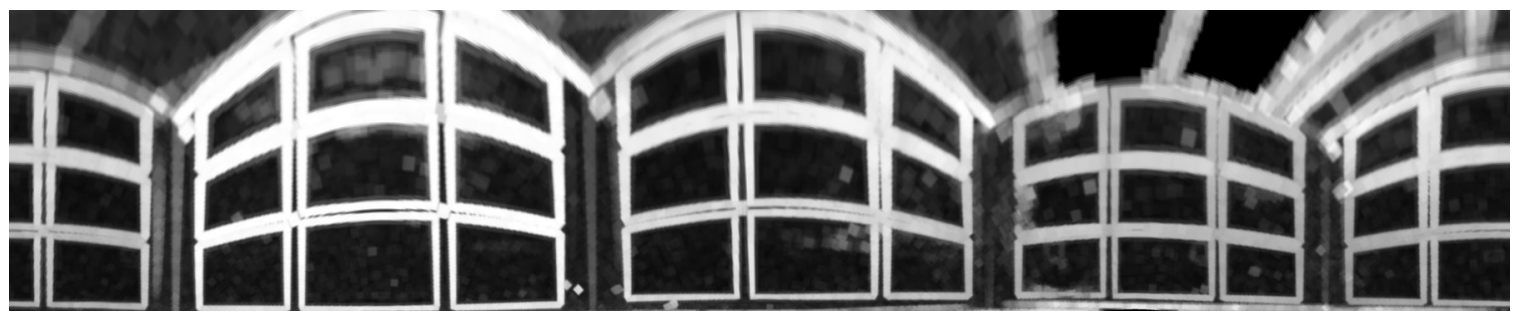

Figure 33: Anti-anamorphosis of a standard morphological gradient.

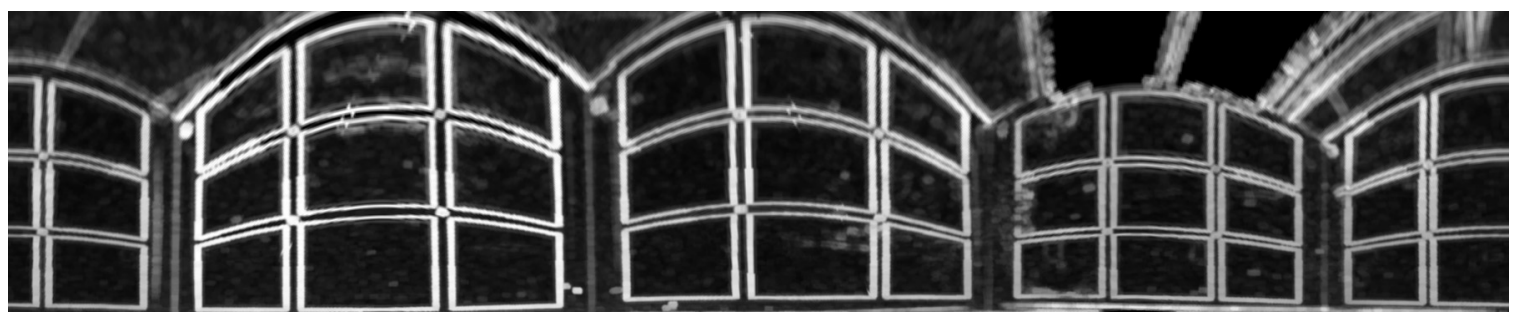

Figure 34: Anti-anamorphosis of an omnidirectional morphological gradient.

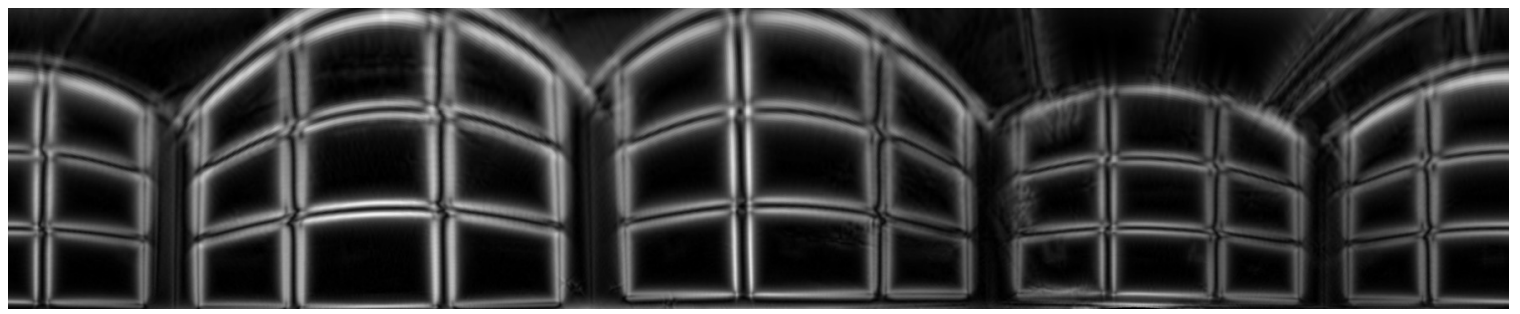

Figure 35: Anti-anamorphosis of a Castan gradient. 


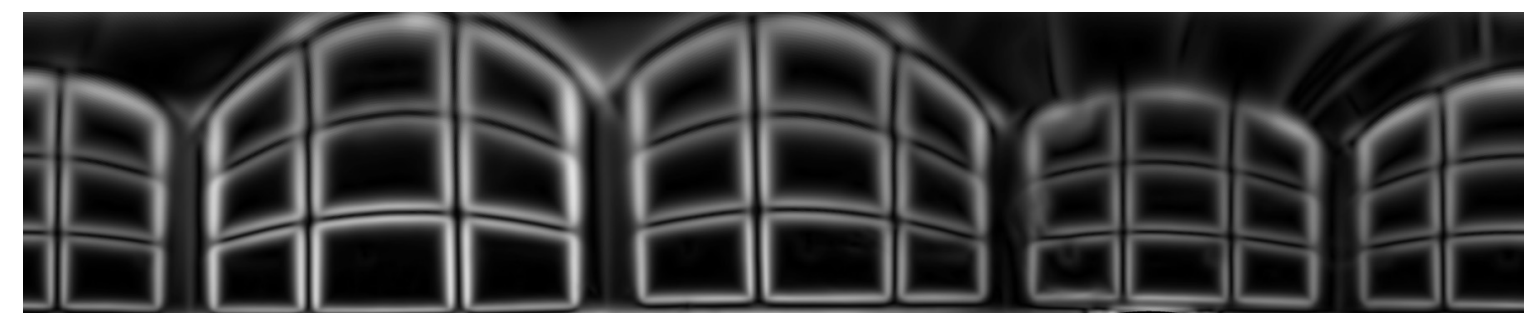

Figure 36: Anti-anamorphosis of a Deriche gradient.

\subsection{Experiment 4: Morphological anti-anamorphosis}

This fourth and last experiment involved anti-anamorphosis of the parabolic image shown in Figure 22, first on the cylinder described in section 2.3, and then on the tangential plane of this cylinder. Figures 37 and 41.a show an example of additive anti-anamorphosis using the interaction matrix described in section 3.7, while Figures 38 and 41.b show the same experiment with non-additive anti-anamorphosis. The gradients mapped to the cases of non-additive anti-anamorphosis are shown in Figures 39 and 41.c. The additive and non-additive anti-anamorphosis results were very comparable. However, on the detailed views in Figure 40, it can be noted that the image obtained by non-additive anti-anamorphosis (Fig. 40.b) is slightly more contrasted than that obtained by additive anti-anamorphosis (Fig. 40.a).

The computation time required by the method using the Choquet integral was about tenfold longer than that of the additive method due to the sorting method involved. Conversely, this trend was reversed when the Sugeno integral was used. It could thus be of interest to implement this image anti-anamorphosis method for realtime anti-anamorphosis applications
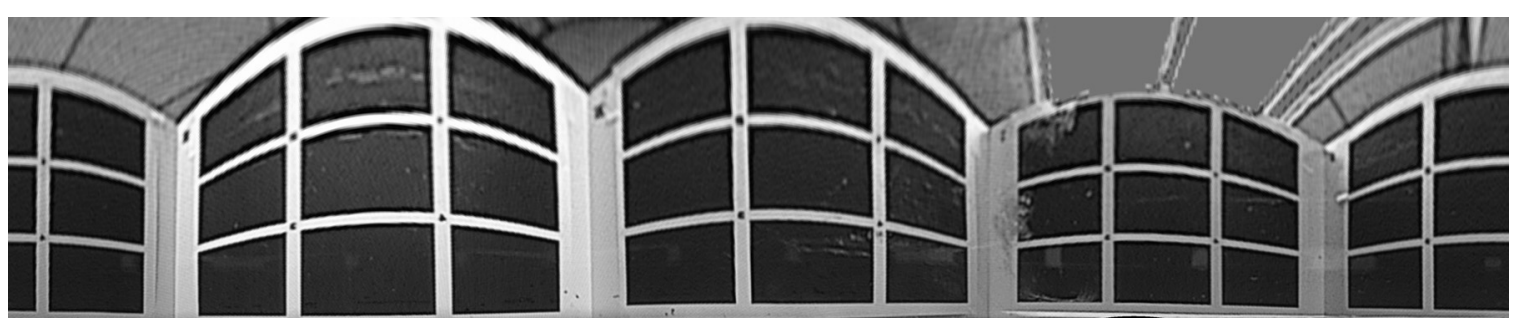

Figure 37: Additive anti-anamorphosis.
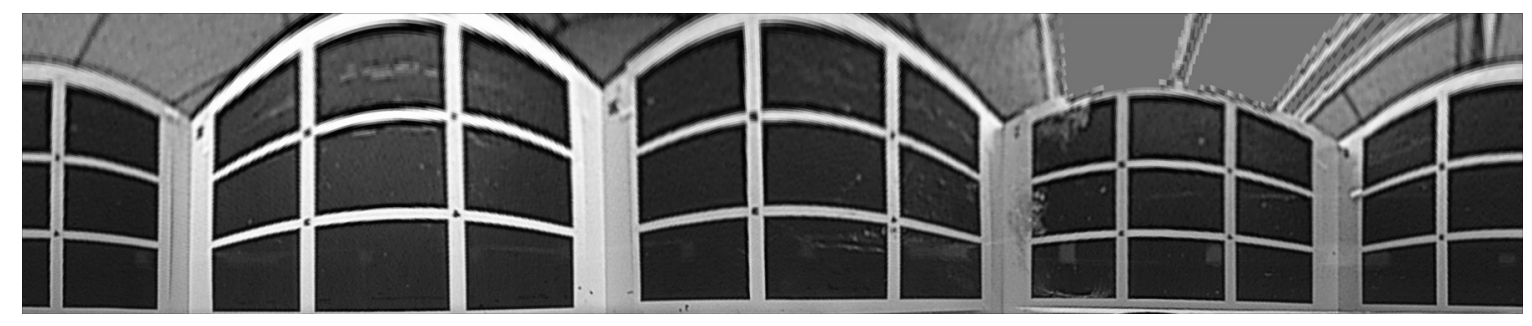

Figure 38: non-additive anti-anamorphosis.

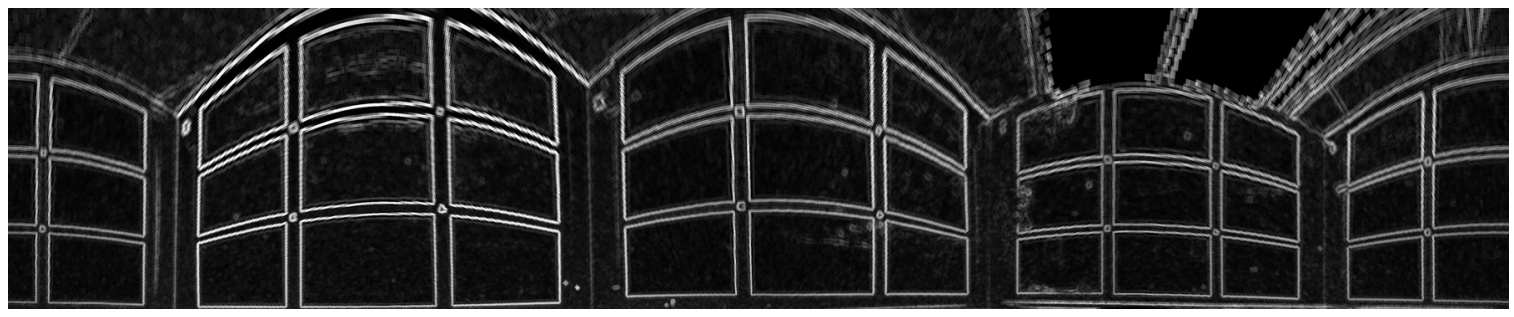

Figure 39: Morphological gradient projected by non-additive anti-anamorphosis. 


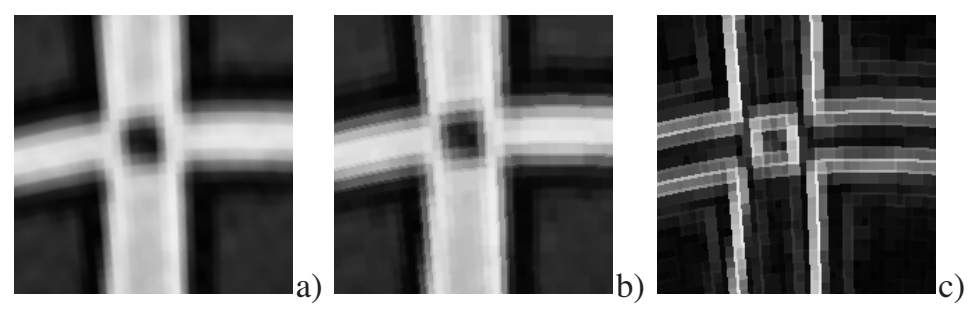

Figure 40: Detailed views: a) additive method, b) non-additive method, c) gradient.
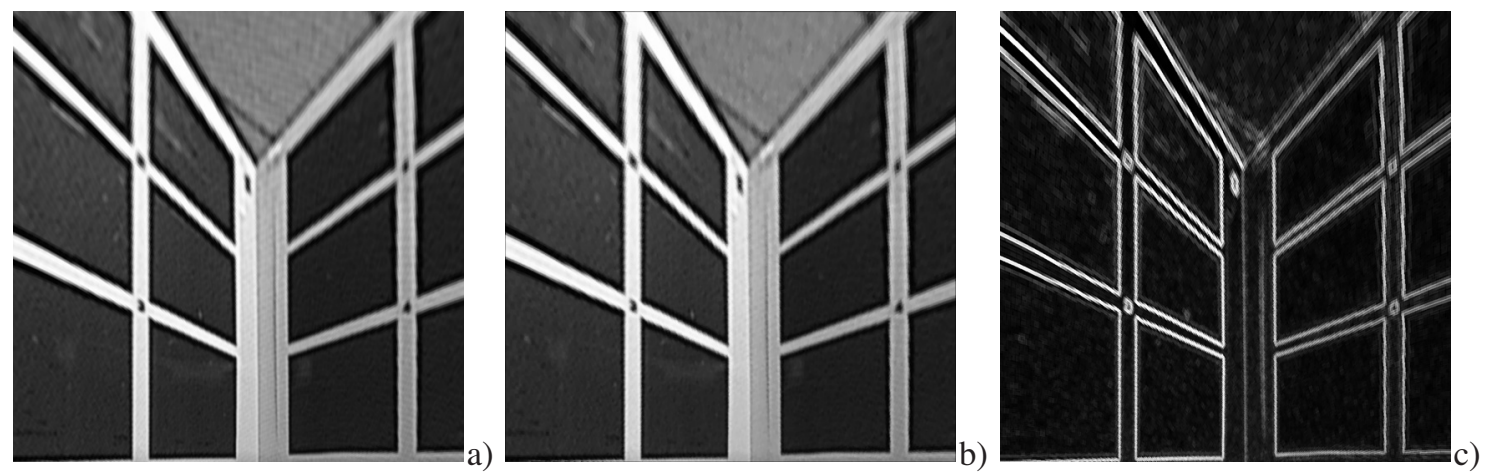

Figure 41: Projective anti-anamorphosis: a) additive, b) non-additive, c) gradient.

\section{Conclusion and Discussion.}

Mathematical morphological tools are capable of effectively analyzing or transforming real projective images when the sensor and objects to be analyzed can be positioned such that a regular mesh on the objects is projected as a regular mash on the image. A morphological operation involving a regular structuring element on the image is thus the projection of a morphological operation equivalent to that which could be achieved on each object.

Risky results may be obtained when using standard morphological tools to analyze images from omnidirectional cameras since there is no relative positioning to overcome all of the deformation effects due to the perspective or anamorphosis induced by this type of sensor.

In this article, we have proposed a new approach to mathematical morphology using the unique features of single viewpoint omnidirectional cameras. This morphological strategy uses a structuring element whose geometry is mapped in a more suitable projective space (here a cylinder surrounding a mirror). This structuring element, when projected on the image, defines a different neighborhood in each pixel of the omnidirectional image. We opted to use a monomodal fuzzy kernel as structuring element to gain awareness on the projected size of objects to be analyzed. Moreover, since we use standard cameras with Cartesian retinas, even binary neighborhoods could not be represented by a union of image pixels. We decided to model sampling phenomena by adopting a strong fuzzy partition to represent a discrete image plane. Most standard summative representations can be generalized by this modeling strategy. It can also avoid generalization of mathematical morphology tools which overlook the discontinuous aspect of illumination information.

The generalization we selected is based on fuzzy integrals. Two methods are presented here-one uses the Choquet integral and the other the Sugeno integral. We decided to only present the Choquet generalization in our illustrated examples in section 4. The Sugeno generalization gave visually comparable results so we felt that it was not worthwhile cluttering the article with more figures.

Operators that do not change the image representation when image filtering is to be done via morphology are very interesting. This is the case for generalization using the Choquet integral, whereby the projected structuring element maps a discrete fuzzy neighborhood and modifies the image grey levels without altering the representation (remaining in the grey level space). However, it has the disadvantage that image grey levels must be sorted, thus complicating computation even though the sorting can be done locally. Moreover, the extension and contraction operators obtained cannot be called dilation and erosion since they do not comply with the union compatibility.

Generalization via the Sugeno integral is in line with all properties of morphological operators and does not require sorting if the possibility level is used as confidence measure. However, by this method, it is not pos- 
sible to take the image grey level directly into account-a normalization step is required, which could be considered as an explicit passage from the grey level space to the fuzzy classification space. This normalization step biases the image interpretation, which can be problematic when morphological tools are to be used for filtering. Local image filtering thus depends on the overall image setting, the sampling base, the image sensor gain and the subjective choice of illumination interpretation.

Our study showed that a structuring element with variable geometry can be used to better account for precognition of projection phenomena that generate omnidirectional images. The method we present can be readily generalized to other projective spaces such as that proposed by Daniilidis. It would now be interesting to conduct a blind comparison of our results with different projective spaces on a very broad range of images.

We showed that it is possible to use projective structuring elements that we developed for anti-anamorphosis operations. Two methods are proposed - a standard method with barycenter estimation, and another method based on an extension of morphological tools. Both methods give visually equivalent results. Computation is very rapid and realtime anti-anamorphosis is possible when a morphological approach based on the Sugeno integral is used. This type of anti-anamorphosis can also automatically generate a gradient image. Finally, the mapping process will be more robust with respect to sampling effects since the grey levels are available in interval format.

Projective structuring elements can also be implemented for statistical filtering. Similarly, this approach can be used to analyze or filter projective images when the projective geometry or an approximate spatial geometry (stereo sensors, structured light vision, images supplemented with distance measures) are known.

The method we present is based on fuzzy integrals, Manhattan distance, T-norm min and the possibility level as confidence measure. It could now be interesting to test other methods such as that proposed by P. Maragos et al. [18,19] or T.-Q. Deng et al. [20], or the different alternative extensions proposed by I. Bloch [2].

Finally, we feel that it would now be essential to investigate a new type of image filtering that would involve non-summative kernels. This strategy could be based on fuzzy integrals with confidence measures (or capacities) other than those used in the present study.

\section{Appendix.}

In this appendix, we have assemble some algorithms that could be used to compute the interaction matrix presented in Section 3.3.

\section{Appendix 1. Dichotomy-based computation of the interaction matrix.}

As presented in Section 3.3.1, the value $\beta_{\mathrm{k}, \mathrm{n}}$ is the highest value that enable an intersection between the $\mathrm{V}\left(\mathrm{P}_{\mathrm{k}}\right)$ and $\mathrm{P}_{\mathrm{n}} \alpha$-level cut. The computation of this value can be simply achieved by a dichotomy-based algorithm. The values of $\Delta \rho$ and $\Delta \theta$ have been assigned to define the projective neighborhood.

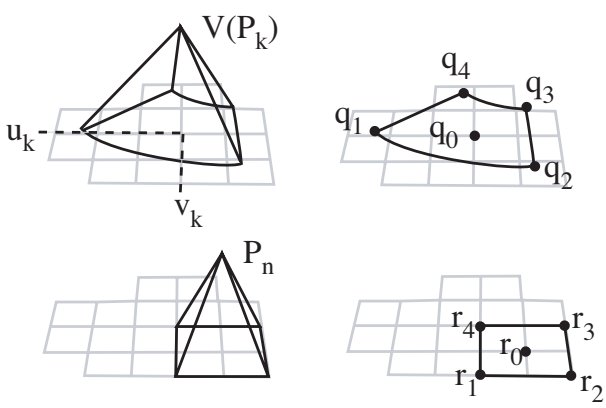

Figure 42: Defining $\mathrm{P}_{\mathrm{n}}$ and approximating $\mathrm{V}\left(\mathrm{P}_{\mathrm{k}}\right)$.

Step 1. If $\mathrm{k}$ equals $\mathrm{n}$ then $\beta_{\mathrm{k}, \mathrm{n}}=1$, end of the computation.

Step 2. Let $\mathrm{q}_{0}$ be the point whose coordinate is $\left(\mathrm{u}_{\mathrm{k}}, \mathrm{v}_{\mathrm{k}}\right)$. Compute $\mathrm{d}$ and $\Delta \mathrm{d}$ with equation (7). Compute $\theta=\operatorname{atan} 2\left(\frac{\left(\mathrm{v}_{\mathrm{k}}-\mathrm{v}_{0}\right)}{\mathrm{k}_{\mathrm{v}}}, \frac{\left(\mathrm{u}_{\mathrm{k}}-\mathrm{u}_{0}\right)}{\mathrm{k}_{\mathrm{u}}}\right)$. Compute the coordinates of the four points $\mathrm{q}_{1 \ldots 4}$ by using the expression

(3) with $\mathrm{d} \pm \Delta \mathrm{d}$ and $\theta \pm \Delta \theta$. Let $\mathrm{r}_{0}$ be the point whose coordinate is $\left(\mathrm{u}_{\mathrm{n}}, \mathrm{v}_{\mathrm{n}}\right)$. Compute the coordinates of the four points $r_{1 \ldots 4}$ (see Fig. 42). Check the intersection between the polygon defined by $q_{1 \ldots 4}$ and the tile 
defined by $r_{1 \ldots 4}$. If the intersection is empty then $\beta_{k, n}=0$, end of the computation.

Step 3. Set $\alpha_{\text {sup }}=1$ and $\alpha_{\text {inf }}=0$.

Step 4. Compute $\alpha=\frac{\alpha_{\text {inf }}+\alpha_{\text {sup }}}{2}$. Define $r_{i}^{\prime}=\alpha r_{0}+(1-\alpha) r_{i}$ and $q_{i}^{\prime}=\alpha q_{0}+(1-\alpha) q_{i}$ for $i=1 \ldots 4$.

Check the intersection between the polygon defined by $\mathrm{q}_{1 \ldots 4}^{\prime}$ and the tile defined by $\mathrm{r}_{1 \ldots 4}^{\prime}$. If the intersection is empty, then set $\alpha_{\text {sup }}=\alpha$ else set $\alpha_{\text {inf }}=\alpha$.

Step 5. If $\left(\alpha_{\text {sup }}-\alpha_{\text {inf }}\right)<$ precision then set $\beta_{k, n}=\alpha_{\text {sup }}$, else go to Step 4 .

Appendix 2. Simplified computation of the interaction matrix.

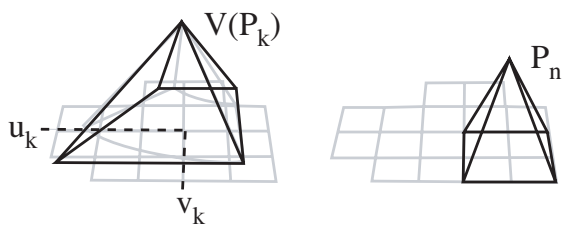

Figure 43: Simplification of $\mathrm{V}\left(\mathrm{P}_{\mathrm{k}}\right)$.

Within this simplification, the neighborhood $\mathrm{V}\left(\mathrm{P}_{\mathrm{k}}\right)$ is said to be the pyramidal fuzzy subset whose mode is $\left(\mathrm{u}_{\mathrm{k}}, \mathrm{v}_{\mathrm{k}}\right)$ and spread is $[\mathrm{du}, \mathrm{dv}]=\left[\mathrm{k}_{\mathrm{u}}(|\mathrm{d} \sin \theta \Delta \theta|+|\cos \theta \Delta \mathrm{d}|), \mathrm{k}_{\mathrm{v}}(|\mathrm{d} \cos \theta \Delta \theta|+|\sin \theta \Delta \mathrm{d}|)\right] . \mathrm{P}_{\mathrm{n}}$ is the pyramidal fuzzy subset whose mode is $\left(\mathrm{u}_{\mathrm{n}}, \mathrm{v}_{\mathrm{n}}\right)$ and spread is $[1,1]$. Let $\Delta \mathrm{u}=\left|\mathrm{u}_{\mathrm{k}}-\mathrm{u}_{\mathrm{n}}\right|$ and $\Delta \mathrm{v}=\left|\mathrm{v}_{\mathrm{k}}-\mathrm{v}_{\mathrm{n}}\right|$. The computation of $\beta_{\mathrm{k}, \mathrm{n}}$ is given by:

$\beta_{\mathrm{k}, \mathrm{n}}=\Pi\left(\mathrm{V}\left(\mathrm{P}_{\mathrm{k}}\right) ; \mathrm{P}_{\mathrm{n}}\right)=\min \left(\alpha_{\mathrm{u}}, \alpha_{\mathrm{v}}\right)$,

with $\alpha_{\mathrm{u}}=\min \left(1, \frac{\Delta \mathrm{u}}{1+\mathrm{du}}\right)$ and $\alpha_{\mathrm{v}}=\min \left(1, \frac{\Delta \mathrm{v}}{1+\mathrm{dv}}\right)$.

\section{Acknowledgments.}

The authors are indebted to El Mustapha Mouaddib for the calibrated parabolic omnidirectional images and Vincent Fournier for the rotational panoramic image used in this article. This work has been partially supported by the CNRS funding.

\section{References.}

[1] J. Serra, Image analysis and mathematical morpholgy, Academic Press Inc., London 1982.

[2] I. Bloch, H. Maître, Fuzzy mathematical morphologies: a comparative study, Pattern Recognition, Vol. 28, \#9, (1995) 1341-1387.

[3] K. Daniilidis, A. Makadia, T. Bülow, Image processing in catadioptric planes: spatiotemporal derivatives and optical flow computation, Proceedings of Omnivis 2002, IEEE Workshop on Omnidirectional Vision, Copenhagen, Denmark, (2002) 3-26.

[4] S. Ieng, R. Benosman, J. Devars, An efficient dynamic multi-angular feature points matcher for catadioptric views, Proceedings of Omnivis 2003, Workshop on Omnidirectional Vision and Camera Networks, USA, Madison Wisconsin (2003).

[5] S. Nayar, Omnidirectional Vision, Proc. of Eight International Symposium on Robotics Research (ISRR), Shonan, Japan (1997).

[6] S. Peleg and M. Ben-Ezra, Stereo panorama with a single camera, Proc. Computer Vision and Pattern Recognition Conf., (1999) 395-401, .

[7] Harsh Nanda, Ross Cutler, Practical Calibrations for a real-time digital omnidirectional camera, Technical Sketches, Computer Vision and Pattern Recognition, Hawaii, US (2001).

[8] Baker S. and Nayar S. K. Single viewpoint catadioptric camera, Panoramic vision: sensor theory and application, monographs in computer science chapter 4, 39-72. Springer-Verlag, New York, 2001 
[9] R. Swaminathan, M. D. Grossberg and S. K. Nayar, Non-Single Viewpoint Catadioptric Cameras: Geometry and Analysis, Columbia University TR, CUCS-004-01, 2001.

[10] C. Geyer, K. Daniilidis, Catadioptric projective geometry, International Journal of Computer Vision, \#43, (2001) 223- 243.

[11] O. Faugeras, Three-Dimensional Computer Vision: a Geometric Viewpoint, MIT press, 1993.

[12] D. Dubois, L. Foulloy, G. Mauris, H. Prade, Probability-possibility transformation, triangular fuzzy sets, and probabilistic inequalities, Reliable Computing, Vol. 10, (2004) 273-297.

[13] D. Demigny, On optimal linear filtering for edge detection, IEEE Transactions on Image Processing, Vol. 11, Issue 7, (2002), $728-737$.

[14] J.-L. Marichal, An axiomatic approach of the discrete Choquet integral as a tool to aggregate interacting criteria, IEEE Transactions on Fuzzy Systems, Vol. 8 \#6, (2000) 800-807.

[15] F. Comby, O. Strauss, M. Aldon, Fuzzy approach of motion estimation, Actes de Fuzz'ieee01: 10 IEEE International Conference on Fuzzy Systems, Melbourne, Australia,(2001).

[16] D. Dubois, H. Prade, Possibility theory: an approach to computerized processing of uncertainty, Plenum Press, London, 1985.

[17] A. Chateauneuf, J. Jaffray, Some characterizations of lower probabilities and other monotone capacities through the use of Mœbius inversion, Mathematical Social Sciences, Vol. 17, (1989) 263-283.

[18] P. Maragos, V. Tzouvaras, G. Stamou, "Synthesis and applications of lattice image operators based on fuzzy norms", Proc. Int'l Conf. Image Processing (ICIP-2001), Thessaloniki, Greece, (2001) 521-524.

[19] P. Maragos and R.D. Ziff, Threshold superposition in morphological image analysis systems, IEEE Transactions on Pattern Analysis and Machine Intelligence, Vol. 12, (1990) 498-504.

[20] T. Deng and H. Heijmans, Grey-scale morphology based on fuzzy logic, J. Math. Imaging Vision 16 (2), (2002) 155-171. 